\title{
II. Private Kultur oder öffentlicher Kultus - Jüdische Religion im nationalsozialistischen Deutschland und im autoritären Polen
}

\section{Zwischen den Traditionen}

\section{Gemeinden und Gemeindemitglieder}

Jüdische Immigranten, die im Laufe des Ersten Weltkrieges aus Osteuropa nach Deutschland gekommen waren, hatten in der Regel einen religiös-orthodoxen Hintergrund. Die Immigration führte im allgemeinen zu Veränderungen des Lebenswandels. Um sich dem Lebensstil in Deutschland anzupassen, gaben viele osteuropäische Einwanderer im Laufe der Zeit die strikt orthodoxe Lebensführung auf1. An einem traditionell-jüdischen Leben, das rituelle, individuelle und gemeindebezogene Aspekte des religiösen Glaubens miteinander verknüpfte, hielten sie jedoch zumeist weiterhin fest. Diese Formen religiöser Praxis unterschieden sich grundsätzlich von dem privaten, häuslich zurückgezogenen Charakter religiösen Lebens deutscher Juden, und so konnten ausländische Juden eben an ihrer Lebensführung leicht als Juden erkannt werden.

Die deutsche Gesellschaft fühlte sich durch fremdartige Sitten und Gebräuche osteuropäischer Juden oft provoziert. Zu Spannungen kam es unter anderem wegen der vielen kleinen Gebetshäuser, die bisweilen in Privatwohnungen großer Mietshäuser untergebracht waren. Beispielsweise wandten sich Nachbarn in Leipzig mit der Forderung an die Polizei, eine Betstube in ihrem Wohngebäude zu schließen. In der Argumentation hieß es, eine Organisation, und noch dazu eine jüdische, bei deren Mitgliedern es sich vorwiegend um Polen handele, könne ohne Registrierung wohl kein Gastrecht in Leipzig beanspruchen². In Hamburg schwankte die Leitung der jüdischen Gemeinde, ob sie ein in ihrem Besitz befindliches Gebäude der osteuropäischen Organisation Adass Jeshurun zur Verfügung stellen solle oder nicht. Die Vertreter der Gemeinde befürchteten eine „judenfeindliche“ Haltung der Nachbarn und forderten Rechenschaft, welche Schritte Adass Jeshurun zu unternehmen gedenke, um eine Störung der Nachbarn zu vermeiden ${ }^{3}$.

Derartig versteckte Zeichen der Ablehnung nahmen infolge der Machtzunahme der Nationalsozialisten offene, gewalttätige Formen an. In der Weimarer Republik war es die Polizei gewesen, die die religiösen Organisationen unter ihren Schutz stellen und ihre Aktivitäten ermöglichen mußte, sofern es sich um gesetzlich aner-

1 Breuer, Jüdische Orthodoxie, S. 58; Heid, Ostjüdische Arbeiter, S. $517 \mathrm{ff}$.

2 StA Leipzig, PP-V 3070, RA Rudolf Jesch an das Polizeipräsidium Leipzig, 17. 6. 1925.

3 StA Hamburg, Deutsch-israelitische Gemeinde in Hamburg, Sitzungprotokolle des Gemeindevorstandes am 4. 8. 1929, 5. 8. 1930. 
kannte Gruppierungen handelte ${ }^{4}$. Ob die deutschen Polizisten allerdings Verständnis für die Menschen hatten, zu deren Schutz sie angefordert wurden, ist zu bezweifeln ${ }^{5}$. Die Machtübernahme der NSDAP ließ das Gefühl der Fremdartigkeit in offene Feindschaft umschlagen und ebnete den Weg für gewaltsame Angriffe auf jüdisch-religiöse Symbole und ihre Träger. So riß die Polizei in Dresden bei einer Leibesvisitation einem Juden den als Untergewand getragenen kleinen Gebetsmantel vom Körper6. Mitglieder der NSDAP in Dresden schlossen einen polnischen Juden in einen Keller ein, rissen ihm die Schnurrbarthaare aus, schnitten ihm den Kinnbart $\mathrm{ab}$ und rasierten sein Haar in der Form eines Hakenkreuzes ${ }^{7}$. In Mannheim stürmten NSDAP-Mitglieder die Synagoge „Shomrei Shabbat" und zerstörten oder entwendeten Kultgegenstände. Nachdem die Parteiangehörigen verschwunden waren, erschienen weitere Nationalsozialisten in Uniform, die die auf dem Boden zerstreuten Heiligen Schriften mit Füssen traten ${ }^{8}$. Dieses Verhalten der Uniformierten regte Nachahmer an. So versammelten sich Straßenverteiler des Stürmers am Freitagabend vor Synagogen polnischer Juden, belästigten die Gottesdienstbesucher und überfielen sie auf dem Heimweg9. Derartige Zwischenfälle weckten bei manchen die Erwartung, man könne sich angesichts der politischen Veränderungen der polnischen Synagogen entledigen. So beschwerte sich eine Anwohnerin in einem Leserbrief an den Stürmer über eine Synagoge, die an Feiertagen und am Schabbat im Waschkeller ihres Wohnblocks eingerichtet werde, wodurch die Hausfrauen an der Benutzung der Waschräume während dieser Tage gehindert würden. Ein jeder, so hieß es in dem Brief, „könne Zeuge dieses jüdischen Gemauschels sein, wenn die jüdischen Familien ... mit Kind und Kegel dorthin strömen. Was sagst Du dazu, lieber Stürmer, und was würde der Führer Adolf Hitler dazu sagen, wenn er diese Zustände mit ansehen müßte. "10 Das religiöse Leben osteuropäischer Juden stellte für die nationalsozialistischen Behörden, aber ohne Frage auch für breite Teile der Bevölkerung, eine Provokation dar.

Angesichts dieser feindseligen Haltung sahen sich die altansässigen jüdischen $\mathrm{Ge}$ meinden gezwungen, ihre Politik gegenüber den separaten religiösen Institutionen osteuropäischer Juden neu zu formulieren. Die Kreise der deutschen Orthodoxie, deren Mitgliederzahlen im Schwinden waren, hatten durch die Emigranten aus Osteuropa in den zwanziger Jahren neuen Zuwachs erhalten ${ }^{11}$. Diese neuen Mitglieder

${ }^{4}$ So wandte sich der Verein jüdischer Händler und Reisender in Leipzig an die Polizei mit der Bitte, eine Veranstaltung des Vereins unter Polizeischutz zu stellen, da „noch heute die Atmosphäre der Andersdenkenden ziemlich gespannt ist". - StA Leipzig, PP-V 4455, Bestand Verein jüdischer Händler und Reisender an das Polizei-Präsidium, 25. 11. 1931.

5 Wie tief Unverständnis und Spott reichten, geht aus dem Bericht eines deutschen Polizisten über eine Sitzung des Vereins Ahavat Thora in Leipzig hervor: StA Leipzig, PP-V 4407, Polizeipräsidium Leipzig Abt. IV, Bericht, 2. 12. 1934.

6 AAN, Amb Rp w Berlinie, Akt Nr. 864, Dr. Brzezinski, Konsul der Republik Polen, an das Ministerium für Auswärtige Angelegenheiten in Dresden, 28. 3. 1933.

7 AAN, Amb Rp w Berlinie, Akt Nr. 864, Dr. A. Kruczkiewicz, Konsul der Republik Polen, an den Polizeipräsidenten, April 1933.

8 AAN, Amb Rp w Berlinie, Akt Nr. 865, Dr. Adam Lisiewicz, Konsul der Republik Polen, an das Badische Staatsministerium in Karlsruhe, 21. 4. 1933.

9 AAN, Amb Rp w Berlinie, Akt Nr. 870, Das polnische Konsulat in Essen an den Polizeipräsidenten in Köln, 31. 7.1935.

10 StA Leipzig, PP-V 4437, Anny Hoffmann an den Stürmer, 30. 10. 1936.

11 Ben-Avner, Vom orthodoxen Judentum, S. $10 \mathrm{f}$. 
beanspruchten zwar in hohem Maße die bestehenden religiösen Dienstleistungen der Orthodoxie, hatten parallel jedoch auch eigene Institutionen errichtet ${ }^{12}$. So blieben also die Beziehungen zwischen der alten deutschen Orthodoxie und den osteuropäischen Juden, insbesondere aus Polen, distanziert ${ }^{13}$. Dies war auch eine Folge des verbreiteten Gefühls der Fremdartigkeit, das die alteingesessene Orthodoxie in Deutschland gegenüber dem religiösen Leben der Juden in Polen bereits vor der Emanzipation gehegt hatte und das nach der Emanzipation natürlich keineswegs geringer geworden war. Das Aufeinandertreffen der deutschen Orthodoxie und der Einwanderer aus Polen weckte Befürchtungen, die polnischen Einwanderer könnten kein Verständnis für den „goldenen Mittelweg“ der modernen Orthodoxie zeigen und würden die religiöse Lebensweise ganz ablegen in der Annahme, diese sei mit den durch die Immigration hervorgerufenen wirtschaftlichen Zwängen nicht vereinbar. Bewahrten die Einwanderer jedoch die religiösen Sitten und Gebräuche ihrer Herkunftsländer, so wurde dies von der deutschen Orthodoxie ebenso negativ beurteilt ${ }^{14}$.

Dennoch war die deutsche Orthodoxie den Einwanderern gegenüber im Prinzip positiv eingestellt - und zwar, weil sie auf neue Mitglieder hoffte bzw. fürchtete, osteuropäische Juden könnten die bestehenden Gemeinden verlassen und separate Gemeinden gründen ${ }^{15}$. In der Frankfurter Austrittsgemeinde war z. B. in den zwanziger Jahren ein Rabbiner ungarischer Herkunft in das Rabbineramt berufen worden, nachdem über die Nominierung Rabbiner Raphael Breuers keine Übereinstimmung hatte erzielt werden können ${ }^{16}$. In der Frankfurter Jeschiwa war die Mehrheit der Schüler osteuropäischer Herkunft. Deutsche Schüler waren dagegen in der Minderheit. Ihre Studiendauer war im allgemeinen nur kurz ${ }^{17}$. Darin zeigte sich eine gewisse Abhängigkeit der deutschen Orthodoxie und ihrer Institutionen von Juden aus Osteuropa.

Das Verhältnis osteuropäischer Einwanderer zum nicht-orthodoxen, deutsch-jüdischen Milieu war seit den zwanziger Jahren durch konstante Spannungen geprägt, zu denen es infolge einiger Grundsatzfragen gekommen war: Gegenstand der Debatte waren die Entwicklung eines religiösen Ritus, der sowohl den Bedürfnissen deutscher Juden als auch denen der Einwanderer entsprach, die Gründung separater osteuropäisch-religiöser Institutionen und deren Beziehungen zu den Gemeinden sowie allgemeine Fragen der Finanzierung und Legitimierung ${ }^{18}$. Praktisch kam es in den dreißiger Jahren zu keinen wesentlichen Veränderungen in der Dynamik, die für die religiösen Beziehungen zwischen den nicht-orthodoxen deutschen Juden und den Einwanderern schon in den zwanziger Jahren typisch gewesen war. Dies geht eindeutig aus der relativ geringen überlieferten Dokumentation hervor. Infolge

12 Munk, Austrittsbewegung, S. $143 \mathrm{ff}$.

13 Breuer, Jüdische Orthodoxie, S. $57 \mathrm{ff}$

14 Beispiele dazu bei: Maurer, Ostjuden, S. 602 f.

15 Maurer, Ostjuden, S. 593 f.; Breuer, Jüdische Orthodoxie, S. $58 \mathrm{f}$.

16 Jakob Katz, der in jenen Jahren in der Jeschiwa in Frankfurt studierte, beschricb, wic schwierig es war, einen Kandidaten zu finden, dessen Allgemeinbildung den hohen Ansprüchen und Normen innerhalb der deutschen Orthodoxie entsprach. Die erfolgte Wahl stellte sich dann als Irrtum heraus, siehe: Katz, Autobiographie, S. $73 \mathrm{ff}$.

17 Katz, Autobiographie, S. 64; IfCJ 11 (32), Wolf Bodenheimer, 23. 9. 1963, S. 3-4.

18 Maurer, Ostjuden, S. $587 \mathrm{ff}$. 
der allgemeinen Verschlechterung der wirtschaftlichen Situation jüdischer Gemeinden im nationalsozialistischen Deutschland wurde auch das Budget der osteuropäisch-religiösen Institutionen von Jahr zu Jahr geringer. Doch schon gegen Ende der zwanziger Jahre waren die Finanzen dieser Gemeinden knapp gewesen, und bereits zu diesem Zeitpunkt war es zu etlichen Einsparungsmaßnahmen gekommen ${ }^{19}$. In den dreißiger Jahren übte z.B. die Hamburger Gemeinde wachsenden Druck auf die osteuropäischen Synagogen aus, um diese von einer notwendigen Kürzung ihrer Ausgaben zu überzeugen ${ }^{20}$, und zeigte großes Interesse an der zahlenmäßigen Reduzierung dieser Synagogen ${ }^{21}$. Doch von den finanziellen Schwierigkeiten waren eben nicht nur osteuropäische Gemeindeinstitutionen betroffen; vielmehr wurde darüber hinaus auch die Zusammenlegung angesehener, traditionsreicher religiöser Einrichtungen des deutschen Judentums wie z.B. der Rabbinerseminare in Berlin und Breslau erwogen 22 .

Der besondere Charakter der Beziehungen zwischen deutschen Behörden, jüdischen Gemeinden und osteuropäischen Juden nach 1933 läßt sich durch die Ereignisse in der Leipziger Gemeinde anschaulich illustrieren. Dieser Gemeinde gehörten verhältnismäßige viele ausländische Juden an. Nach statistischen Angaben, die 1938 von Fritz Arlt ${ }^{23}$ im Auftrag der Nationalsozialisten und in deren Interesse erhoben wurden, ergibt sich folgendes Bild: der Anteil deutscher (männlicher) Juden in Leipzig an der Zahl jüdischer Männer insgesamt lag bei 38,6\% gegenüber einem prozentualen Anteil von 61,4\% ausländischer jüdischer Männer ${ }^{24}$. Der prozentuale Anteil deutsch-jüdischer Männer entsprach dem der polnisch-jüdischen (38,8\%). Von ungefähr 11000 Juden, die zum Zeitpunkt der Erhebung in Leipzig wohnten, waren 4019 außerhalb der deutschen Länder, hauptsächlich in Polen (3084), geboren worden. Die Zahl der Personen ohne Staatsbürgerschaft oder mit ungeklärter Staatsbürgerschaft lag bei 1500, wobei anzunehmen ist, daß auch diese Gruppe sich überwiegend aus Juden polnischer Herkunft zusammengesetzt haben dürfte. Der Geburtsort der übrigen jüdischen Einwohner Leipzigs, die nicht aus Deutschland oder Polen stammten, lag im allgemeinen ebenfalls in Osteuropa.

Unter den in Leipzig herrschenden besonderen Bedingungen war es nur natürlich, daß die Führung in allen Fragen des religiösen Charakters der Gemeindeinstitutionen bei den osteuropäischen Juden lag. Im Erziehungssektor reichte die Zahl der liberalen deutschen Juden nicht aus, um eine eigene Schule aufrechtzuerhalten, so daß Kinder liberaler Familien auf orthodoxe Schulen geschickt wurden ${ }^{25}$. Auf-

19 StA Hamburg, Deutsch-Israelitische Gemeinde in Hamburg, Sitzungsprotokolle des Repräsentantenkollegiums nebst Tagesordnung 12. 2.1929-22. 12. 1932, 360a.

20 So wurde der "Talmud-Thora“ dic Neubesetzung der Rabbinatsstelle verweigert, nachdem der Amtsinhaber verstorben war: StA Hamburg, Deutsch-Israelitische Gemeinde in Hamburg, Sitzungsprotokolle des Repräsentantenkollegiums, Etatberatungen für 1936; Sitzungsprotokolle des Gemeindevorstandes, 2. 2. 1937.

21 Vgl. z. B. StA Hamburg, Deutsch-Israelitische Gemeinde in Hamburg, Sitzungprotokolle des Repräsentantenkollegiums, Etatberatungen für 1936.

22 CZA, A 142 90/4a, Brief des Kuratoriums der Kommerzienrat Fränckel'schen Stiftungen an Alfred Klee, 28. 10. 1936.

23 Zu Arlts „wissenschaftlicher Karriere“ siehe: Aly/Heim, Vordenker der Vernichtung, S. 168.

${ }_{24}$ Arlt, Volksbiologische Untersuchungen, S. 38.

25 StA Leipzig, PP-V 4437, Polizeipräsidium Leipzig, Überwachungsbericht - Israelitische Religionsgemeinde zu Leipzig, 22. 4. 1936. 
grund der Zahlenverhältnisse gab es in Leipzig unzählige Synagogen und Gebetshäuser, die den Bedürfnissen kleiner Gruppen osteuropäischer Gläubiger nach Herkunftsland oder Wohngegend entsprachen. Die nationalsozialistischen Behörden betrachteten diese Verhältnisse mit Unwillen. Vor den jüdischen Feiertagen im Jahre 1936 informierte die Leipziger Polizei die jüdische Gemeinde, sie könne keinen ausreichenden Schutz vor allen in der Stadt zerstreuten Synagogen aufstellen. Die Gemeinde wurde aufgefordert, sich um die zahlenmäßige Reduzierung der Gebetsstätten zu kümmern ${ }^{26}$. Dieses Verhalten der Polizeibehörden war typisch für etliche Widersprüche in der nationalsozialistischen Politik: 1936 wurden Juden durch eine diskriminierende Gesetzgebung in allen Bereichen hart getroffen, gleichzeitig aber sahen sich die Behörden verpflichtet, die Stätten, an denen sich Juden während der Feiertage trafen, zu schützen. Die Gemeindevertreter erklärten zwar, auch sie hätten ein grundsätzliches Interesse an der quantitativen Reduzierung der Synagogen, bezweifelten jedoch, ob dies praktisch durchführbar sei. Die osteuropäischen Juden, so betonten sie, mieden die große Synagoge, die ihnen unattraktiv erscheine, und seien außerdem auf die Gebetshäuser in ihrer Wohngegend angewiesen, da sie an Schabbat und Feiertagen keine Verkehrsmittel benutzten. Die Gemeinderepräsentanten verwiesen darauf, daß es schließlich auch in der Grenadierstraße des Berliner Scheunenviertels zahlreiche kleine Gebetshäuser gebe. Die Polizei begnügte sich 1936 noch mit derartigen Erklärungen und ging nicht gegen die Synagogen vor. Allerdings forderte sie von der Gemeindeleitung, die Synagogenzahl bis zum nächsten Jahr zu reduzieren.

In der Zwischenzeit wandte sich die Leipziger Polizei an die Preußische Geheime Staatspolizei in Berlin und erfuhr dort, daß den 153000 Mitgliedern der dortigen jüdischen Gemeinde 67 Synagogen zur Verfügung stünden: 17 unterstanden der jüdischen Gemeinde, bei den restlichen 50 handelte es sich um private Synagogen ${ }^{27} . \mathrm{Zu}$ den Feiertagen kämen weitere 20 private Gebetshäuser in verschiedenen Sälen und Räumlichkeiten hinzu. In Leipzig gab es dagegen polizeilichen Quellen zufolge für 11500 Menschen 13 große und kleine Synagogen sowie vier weitere Gebetsstuben in Privatwohnungen und -zimmern ${ }^{28}$. Diese Informationen führten die Behörden offensichtlich zu dem Schluß, dic Zahl der Synagogen in Leipzig sei zu hoch. In der Folge forderte die sächsische Landesregierung die Geheime Staatspolizei auf, die Tätigkeit so vieler Synagogen in Leipzig zu verbieten. Die Polizei bestand darauf, daß alle Synagogen, die sich in einem unwürdigen $Z u$ ustand befanden, zu verschwinden hätten ${ }^{29}$.

Der jüdische Gemeindevorstand in Leipzig hatte sich, wie erwähnt, 1936 verpflichtet, für eine Reduzierung der Synagogenzahl zu sorgen. Dabei hatte er betont, dies liege im eigenen Interesse der jüdischen Gemeinde. Vermutlich hatte der Gemeindevorstand hier auf diesem Wege den Behörden zuvorkommen und den Anschein einer gegen die osteuropäischen Juden gerichteten Zusammenarbeit erwekken wollen. Doch im Sommer 1937 sah sich der Vorstand erneut mit der polizeili-

26 StA Leipzig, PP..V 4437, Polizeipräsidium Leipzig, Abt. 17, 1. 6. 1936.

27 StA Leipzig, PP-V 4437, Preußische GeStaPo an den Polizeipräsidenten in Leipzig, Überwachungsbericht - Israelitische Religionsgemeinde zu Leipzig, 30. 9. 1936.

28 StA Leipzig, PP-V 4437, Polizeilicher Bericht an den Polizcipräsidenten, 8. 9. 1936.

29 StA Leipzig, PP-V 4437, Regierungsassessor an die GeStaPo, 8. 4. 1937. 
chen Forderung konfrontiert, seiner Verpflichtung nachzukommen. Nun aber verteidigte er nachdrücklich die Existenzberechtigung der Synagogen. Nach einer Besichtigung aller Synagogen teilte der Gemeindevorstand mit, alle Bethäuser seien in einem durchaus würdigen und vollständig sauberen Zustand befunden worden. Keines der Bethäuser liege unmittelbar an der Straße. Alle scien als Bethäuser deutlich erkennbar, so daß jegliche Mißdeutung der Gebetsversammlungen durch Nachbarn ausgeschlossen $\operatorname{sei}^{30}$. Alle Gebetshäuser seien zu den Feiertagen gut besucht. Es könne daher zu einer Überfüllung der kleinen Synagogen kommen, wenn die Polizei ihre Ankündigung wahr mache und zwei der größeren Synagogen der Gemeinde schließen lasse. Außerdem sah sich der Vorstand nicht in der Lage, die verschiedenen Liturgien der Gläubigen ganz unterschiedlicher Herkunft zu einem Gebetsritus zu vereinheitlichen. Auch mit einem Appell an das Traditionsempfinden versuchte der Gemeindevorstand, ein Einlenken der Behörden zu erreichen:

„Zu alldem haben die Beter einer Synagoge eine Einstellung selbstverständlicher Pietät gegenüber ihrer ihnen seit Jahren und Jahrzehnten ... ans Herz gewachsenen Gebetsstätte. Sie hängen natürlicherweise an den alten geheiligten Gebetsstätten, an denen sie und schon ihre Väter und Mütter ihre Gebete verrichtet haben."

Leider liegen keine internen Dokumente der jüdischen Gemeinde zu Leipzig vor, aus denen die Motive für diese eindeutige Position hervorgehen. Doch die Korrespondenz zwischen dem jüdischen Gemeindevorstand und der Leipziger Polizei zeigt deutlich, daß der Vorstand dieser Gemeinde mit absoluter osteuropäischer Mitgliedermehrheit sich mit allen Kräften für den Fortbestand der unabhängigen osteuropäischen Synagogen einsetzte ungeachtet der Tatsache, daß der Vorstand selbst mehrheitlich aus deutschen Juden bestand. Die Leipziger Polizei verfolgte weiterhin aufmerksam die Tätigkeit der kleinen Synagogen und schränkte deren Bewegungsspielraum nach und nach ein ${ }^{31}$. Langfristig, und dies war wohl schon von Anfang an abzusehen, hatte die Gemeinde bei dieser Auseinandersetzung keine wirklichen Erfolgschancen. Der letzte Bericht der Leipziger Gemeinde an die Gestapo erfolgte zwei Monate nach Kriegsausbruch, also Ende 1939. Er meldete die Schließung der letzten dieser Synagogen ${ }^{32}$.

\section{Zwischen "Rabbiner" und "Rebbe"}

Seit den Tagen der Aufklärung, ja eigentlich auch schon vorher, war es deutsch-jüdischen Gemeinden schwergefallen, die religiösen Ämter mit einheimischen Juden zu besetzen. Dies hatte in erster Linie an der unzureichenden jüdisch-religiösen Bildung und Ausbildung in Deutschland gelegen, aber auch an den niedrigen, für einheimische Juden wenig attraktiven Gehältern, die den Amtsträgern in der Regel gezahlt wurden. Viele Inhaber eines religiösen Amtes in deutsch-jüdischen Gemeinden kamen daher aus Osteuropa, wo sie ihre religiöse Ausbildung im Geiste der ost-

30 StA Leipzig, PP-V 4437, der Vorstand der Israelitischen Religionsgemeinde Leipzig an die Polizeistelle in Lcipzig, 2. 7. 1936.

31 StA Leipzig, PP-V 4453, GeStaPo am 13. 12. 1938; Bethaus Mischnais an die Polizei, 13. 12. 1938.

32 StA Leipzig, PP-V 4453, Israelitische Religionsgemeinde zu Leipzig an die GeStaPo, 25. 10. 1939. 
europäischen Orthodoxie genossen hatten. Diese Amtsträger gehörten also stets einerseits zu den osteuropäisch-jüdischen Einwanderern in Deutschland, bildeten andererseits aber einen integralen Bestandteil des religiösen Lebens der deutschen Juden ${ }^{33}$. Obwohl das Synagogenleben deutscher Juden also ohne die Anstellung von Rabbinern, Kantoren und Religionslehrern aus Osteuropa im Grunde nicht möglich gewesen wäre, wurde ihre Präsenz insbesondere nach der Aufklärung stets kritisch beurteilt ${ }^{34}$. Negative Darstellungen wurden zu einem charakteristischen Topos der späteren deutsch-jüdischen Forschungsliteratur, so daß wir nur mit Schwierigkeiten den Wahrheitsgehalt derartiger Kritik von den Vorurteilen der Zeitgenossen trennen können ${ }^{35}$.

Auch während der Weimarer Republik waren osteuropäische Juden in den Reihen der religiösen Amtsträger stark vertreten. Teilweise handelte es sich wie in der Vergangenheit um eingewanderte Rabbiner aus Osteuropa. Doch auch in den Rabbinerausbildungsstätten in Deutschland war der Anteil osteuropäischer Juden höher als der deutscher Juden ${ }^{36}$. In manchen Gemeinden stießen die osteuropäischen Amtsträger allerdings auf heftige Ablehnung. So widersetzten sich z.B. liberale Juden und später auch die lokale Orthodoxie in Danzig der Ernennung und Amtsführung des in Wilna geborenen Rabbiners Sagalovich. Sie lehnten seine Predigten in Jiddisch ebenso ab wie seine Verachtung der staatlichen Gesetze. Auf Widerspruch stießen auch die Zahlungen, die er für besondere Dienstleistungen beanspruchen zu können glaubte, seine Großzügigkeit gegenüber Hausierern und Armen und vieles andere an Sitten und Gebräuchen die dem religiösen Leben deutscher Juden völlig fremd waren ${ }^{37}$.

Neben den Anpassungsschwierigkeiten und der bisweilen ablehnenden Haltung der jüdischen Gemeinden mußten sich religiöse Amtsträger aus Osteuropa mit ihrem unklaren rechtlichen Status gegenüber den deutschen Behörden auseinandersetzen, die in der Regel keine eindeutigen Aussagen über den Status des Einwanderers und seines Arbeitsverhältnisses machten. Bis 1926 mußten jüdische Gemeinden in Preußen eine offizielle Arbeitsgenehmigung für die Beschäftigung osteuropäischer Juden in religiösen Ämtern einholen, denn das Beschäftigungsverhältnis war die Grundlage ihrer Einbürgerung, die die Behörden verhindern wollten. Nach 1926 bestand diese Pflicht zur Arbeitsgenehmigung nicht mehr, obwohl die Behörden weiterhin betonten, es bestünde kein Interesse an der Beschäftigung ausländischer Juden. In Sachsen war es bereits 1925 zu einer Verschärfung der Politik hinsichtlich der Beschäftigung von Ausländern gekommen. Da jedoch die juristische Definition der religiösen Ämter nicht eindeutig war, konnte das Gesetz unterschiedlich interpretiert werden: gegen eine Einstellung ausländischer Juden in die-

33 Gierowski, Juden in Polen, S. $15 \mathrm{ff}$.

34 Gotzmann, Rabbiner.

35 Gotzmann führt einige Beispiele an, vgl. u. a. Brann, Geschichte der Juden, S. 69; Freimark, Die Entwicklung des Rabbinats, S. 12; Katz, Rabbi Raffael Cohen, insbesondere S. 248. Gotzmann weist nach, daß bestimmte Sitten und Gebräuche, die dem rabbinischen Einfluß aus Osteuropa zugeschrieben wurden, also z. B. das Barttragen, in gleichem Maße auch unter deutschen Rabbinern üblich und verbreitet waren.

36 Maurer, Ostjuden, S. 607.

37 Stern, Rabbi Sagalovich. 
sen Ämtern - von liberalen Juden - oder aber zugunsten ihrer Beschäftigung - von der Jüdischen Volkspartei ${ }^{38}$.

In den ersten Monaten des Jahres 1933 kam es zu keinen wesentlichen Veränderungen bei der Besetzung von Rabbinatsstellen und anderer religiöser Ämter in den Gemeinden ${ }^{39}$. Anfang Juli 1933 wurde das Gesetz von 1926, das die jüdischen Gemeinden von der Beschaffung einer Arbeitsgenehmigung freigestellt hatte, aufgehoben. Fortan war eine Genehmigung vom Ministerium für Wissenschaft, Kunst und Volkskultur notwendig, um religiöse Ämter mit ausländischen Juden zu besetzen ${ }^{40}$. Bereits längerfristig Beschäftigte benötigten keine Sondergenehmigung, ihre Anstellung fiel in den Rahmen des Gesetzes zur Beschäftigung von Ausländern vom 23. Januar 1933. Unklarheiten über die neuen Gesetzesverschärfungen brachten die jüdischen Gemeinden und religiösen Körperschaften in Verlegenheit, wie aus den internen Beratungen zwischen jüdischen Organisationen und Gemeinden hervorgeht. So wies der Verband jüdischer Gemeinden die Einstellung eines Rabbiners mit Schweizer Staatsbürgerschaft eilig zurück, da er die Konsequenzen scheute, die mit der notwendigen Erklärung eines Mangels an einheimischen Kandidaten verbunden gewesen wären ${ }^{41}$.

Im Vorstand der Hamburger Gemeinde kam es zu einer heftigen Debatte, nachdem bekannt geworden war, daß der Synagogen-Verband das Amt des Oberrabbiners mit einem ausländischen Kandidaten besetzen wollte" ${ }^{42}$. Obwohl diese Angelegenheit nicht in den Zuständigkeitsbereich des Gemeindevorstandes fiel, wurde sie von ihm eingehend erörtert. Ein Teil der Sitzungsteilnehmer trat mit dem scheinbar neutralen Argument für die Wahl eines deutschen Rabbiners ein, dieser könne die Gemeinde besser repräsentieren. Max Warburg meinte im Namen des gesamten Vorstandes, die Ernennung eines nicht-deutschen Rabbiners sei eine fragliche, „sehr gefährliche" Angelegenheit. Die Debatte drehte sich vornehmlich um die Frage, ob ein ausländischer Rabbiner die Gemeinde vor den Behörden vertreten könne. Dr. Isak Unna wandte sich auf der Vorstandssitzung gegen eine derartige Argumentation. Er sei erstaunt über die Art und Weise, mit der der Vorstand die Angelegenheit handhabe. Offenbar herrsche die Ansicht, es müsse ein Unterschied zwischen deutsch und nicht-deutsch gemacht werden. Man müsse darunter einen Strich zie-

38 Maurer, Ostjuden, S. $609 \mathrm{f}$.

39 In einem Einzelfall entschied das preußische Innenminsterium im Mai 1933, den Vorsänger der Breslauer Gemeinde nach 13jähriger Tätigkeit nach Polen auszuweisen. Diese Ausweisung erfolgte jedoch nicht aufgrund der nationalsozialistischen Ideologie, sondern stand in der Kontinuität der preußischen Beamtenroutine: AAN, Amb Rp w Berlinie, Akt Nr. 913, Der Regierungspräsident an das Konsulat der Republik Polen in Breslau am 12. 5. 1933.

40 Informationsblätter (Hilfe und Aufbau), Ausländische Rabbiner, Nr. 8, 1. 8. 1933.

41 CZA, A 142 88/2, Preußischer Landesverband jüdischer Gemeinden an Klee, 14. 12. 1934. Die Frankfurter Gemeinde wandte sich an die Gemeinde zu Breslau, um sich über deren Erfahrungen mit der Beschäftigung von Ausländern informieren zu lassen. Aus Breslau erhielt man die Antwort, daß von den zahlreichen Anfragen der Breslauer Gemeinde an die Behörden nur cin Antrag zurückgewiesen worden sei, jedoch aus Gründen, die mit der Persönlichkeit des Antragstellers zusammenhingen, während man in den meisten Fällen eine positive Antwort oder noch kcine Antwort crhalten hättc: ZIH, Synagogengemeinde zu Breslau, Akt Nr. 128, Der Vorstand der Synagogen-Gemeinde Breslau an den Vorstand der Israelitischen Gemeinde Frankfurt a. M., 3. 9. 1934.

42 StA Hamburg, Deutsch-Israelitische Gemeinde in Hamburg, Sitzungsprotokolle des Repräsentantenkollegiums, Akt 360e 56, Sitzung Juni/Juli 1935. 
hen. Vor Behörden habe der Gemeindevorstand oder der Vorstand des SynagogenVerbandes als Vertreter aufzutreten, ein religiöses Gutachten könne aber auch von einem Ausländer abgegeben werden, wenn er nur ein Kenner der jüdischen Lehre sei. Die Gemeinde brauche einen jüdischen Rabbiner.

Was Warburg und andere veranlaßt haben mag, sich gegen die Ernennung nichtdeutscher Rabbiner auszusprechen, ist nicht zu klären. Es sieht jedoch so aus, als ob einige Gemeinden befürchteten, die Ernennung eines nicht-deutschen Rabbiners könne irgendwelche negativen Folgen haben. So wandte sich die Gemeinde in Oldenburg z. B. mit der Frage an Dr. Rabin, den Leiter des Breslauer Rabbinerseminars, ob er nicht einen konservativen, jüngeren deutschen Kandidaten habe, den er nach Oldenburg empfehlen könne, denn die Bestätigung eines Ausländers erscheine dort ausgeschlossen ${ }^{43}$. Viele Gemeinden dürften sich also aus Furcht vor möglichen Komplikationen gegen die Einstellung eines nicht-deutschen Rabbiners gewandt haben. Es überrascht daher nicht, wenn ein Rabbinatskandidat in seinem Lebenslauf darauf hinwies, er sei seit seiner Geburt im Besitz der preußischen Staatsangehörigkeit ${ }^{44}$.

Die Möglichkeiten osteuropäischer Rabbiner, sich ihren Lebensunterhalt in Deutschland zu verdienen, verringerten sich im Laufe der dreißiger Jahre erheblich. Zum Teil arbeiteten sie ja in kleinen Gemeinden, deren Mitgliederzahlen in diesen Jahren infolge der Binnen- und Auswanderung stark sanken. Darüber hinaus führte auch die Beschäftigungspolitik der Gemeinden zu erheblicher Not unter jenen Rabbinern, die damals mit der Suche nach einem Arbeitsplatz begannen, was nicht wenige ins Ausland führte ${ }^{45}$. Auch dort stießen sie auf unerwartete Schwierigkeiten. Etliche dieser Rabbiner hatten ihre Ausbildung in einem der deutschen Rabbinerseminare erworben, so daß sie nun zwischen alle Kategorien fielen: Sie stammten zwar aus Osteuropa, ihre religiöse Ausbildung war jedoch auf die Richtlinien des deutschen Judentums abgestellt gewesen. So empfahl Dr. Alfred Klee im Jahre 1935 einen Absolventen des Berliner Rabbinerseminars für die Rabbinatsstelle in Czernowitz. In dem Empfehlungsschreiben heißt es u. a.:

„Es handelt sich bei Dr. Berkovitz in charakterlicher Beziehung um einen außerordentlich zu empfehlenden jungen Mann, der gut spricht, tiefe Kenntnisse unseres Schrifttums hat und von den Dozenten des Seminars warm empfohlen wird. (...) Es dürfte auch in weiten Kreisen in Berlin der Wunsch bestehen, ihn dieser Arbeit hier zu erhalten, wenn nicht mit Rücksicht auf seine Ausländereigenschaft eine Anstellung ausgeschlossen erschiene. Seine Dissertation über ,Hume und der Deismus', mit der er an der Berliner Universität doktoriert hat, ist eine gedie-

43 CZA, A 142 90/4a, Brief an Seminardirektor Dr. J. Rabin am 24. 5. 1935. Die Gemeinde wählte im Oktober 1935 Rabbiner Herbst in das Amt des Rabbiners der Stadt. Einige Mo nate später sah sich Herbst jedoch bereits vor der Entlassung mit der Begründung, er sei inzwischen durch Ausbürgerung staatenlos geworden und führe zudem den Doktortitel, obwohl er infolge der Verhältnisse scin Promotionsexamen nicht hatte ablegen können. Seine abgeschlossene Universitätsbildung allein berechtige ihn nicht zum Führen des Doktortitels. - CZA, A 142 55/2, Rabbiner Hoffmann (Frankfurt) an Alfred Klee, 13. 5. 1936.

44 CZA, A 142 88/2, Bernhard Brilling.

45 CZA, A 142 87/11d, Cantor Leo Halbern (Görlitz) an Klee, 27. 1. 1938; A 142 58/3, Klee an Prof. Heinemann, 3. 2. 1938; A 142 87/11a, Ein Brief von Klee an Edinburgh Hebrew Congregation Synagogue, 9. 8.1938. 
gene Arbeit, wie er auf allen Gebieten, auf profanem Gebiet, wie auch auf dem Gebiet der jüdischen Wissenschaft gute Kenntnisse hat." ${ }^{46}$

Klee wollte sich ohne Zweifel nützlich erweisen, doch gerade die Betonung der Allgemeinbildung des Kandidaten erwies sich als Hindernis. Die jüdische Gemeinde in Czernowitz hatte keinerlei Interesse an einem Rabbiner deutschen Stils. So erhielt Klee die Anwort, es gebe in Czernowitz bereits einen Prediger in der Person des Oberrabbiners Dr. Mark. Zu besetzen sei die Stelle eines orthodoxen Rabbiners ostjüdischer Prägung, mit „einen Raw mit Bart und Pejes. Bildung schadet nichts, aber der Doktor ist von Übel“47.

Die liberalen Institutionen der Rabbinerausbildung schienen die Zeichen der Zeit nicht verstanden zu haben. Das Breslauer Rabbinerseminar legte Wert darauf, daß unter allen Umständen die Allgemeinbildung deutscher Rabbiner aufrechterhalten blieb. Ein Niedergang des wissenschaftlichen Niveaus des Seminars sei ebenso zu verhindern wie der Gefahr, daß die ihre Studien in Deutschland absolvierenden Rabbiner innerhalb und außerhalb Deutschlands gar als Halbakademiker dargestellt werden könnten ${ }^{48}$. Allerdings darf bezweifelt werden, ob tatsächlich die umfangreiche Allgemeinbildung das Hauptproblem der deutschen Rabbinerseminarabsolventen in den dreißiger Jahren war. Die Führung der Orthodoxie in Deutschland mußte bald einsehen, daß die wirklichen Probleme in einem völlig anderen Bereich lagen. Die wenigen Rabbiner, die letztlich außerhalb Deutschlands eine Anstellung gefunden hatten, mußten nicht ihre Allgemeinbildung unter Beweis stellen. Vielmehr wurde von ihnen verlangt, daß sie die Sprache ihrer neuen Gemeinden beherrschten und sich geistig auf die Mitglieder der jeweiligen Gemeinden cinstellten, die ja eigentlich einen „Rebbe“ erwarteten ${ }^{49}$.

\section{Die Auseinandersetzungen um das koschere Schlachten}

\section{Deutschland: Die Suche nach einem Hetter}

Im religiösen Bereich waren Juden in Deutschland und Polen nicht nur durch den direkten Kontakt der osteuropäischen Rabbiner mit den deutsch-jüdischen Gemeinden oder den Aufenthalt als Schüler deutsch-jüdischer Herkunft an osteuropäischen Talmudschulen verbunden. Vielmehr verbanden auch die bereits vor dem Zweiten Weltkrieg in Deutschland und Polen gleichermaßen zunehmenden antisemitischen Angriffe das religiöse Leben der Juden in beiden Ländern. Ein eindrückliches Beispiel in diesem Bereich ist die Auseinandersetzung, die um das Schächten (Schechita), also das koschere Schlachten, in beiden Ländern geführt wurde. In dieser Frage beeinflußten sich das nationalsozialistische Deutschland und Polen gegen-

46 CZA, A 142 87/11a, Klee an Dr. Meier Ebner, Cernauti (Czernowitz), 1. 7. 1935.

47 CZA, A 142 87/11a, Dr. Meier Ebner aus Cernauti an Klee, 2. 8. 1935.

48 CZA, A 142 86/13, Protokoll der Sitzung des Ausschusses für Rabbinerfragen, 18. 1. 1937.

49 So z. B. in Amerika: „Ein Bedürfnis nach geistlichen Führern, die weder Jiddisch noch Englisch sprechen, wird in Amerika natürlich nicht empfunden, und die deutschen Kreise, die wohl in steigendem Umfang drüben entstehen werden, haben für die ersten Jahre gewiß noch nicht die Kraft sich geistige Führer zu berufen." - CAHJP, Ansbach N1/48, Jakob Rosenheim an Rabbiner Dr. Pinchas Kohn, 25. 7. 1938. 
seitig. In Deutschland war das koschere Schlachten bereits im April 1933 verboten worden. In Polen erging am 1. Januar 1937 ein teilweises Schächtverbot, doch die öffentliche Diskussion um ein absolutes Schächtverbot verstummte endgültig erst infolge des Kriegsausbruchs. Der Kampf gegen die gesetzlichen Maßnahmen zum Verbot des Schächtens führte zu einer internen Debatte über das Wesen der jüdischen Religion und Lebensweise in Deutschland und Polen.

Schon im 19. Jahrhundert war das koschere Schlachten nach dem jüdischen Religionsgesetz in vielen Ländern Europas angegriffen worden. Die Schweiz hatte bereits 1893 eine Volksbefragung durchgeführt, infolge derer das Schächten für ungesetzlich erklärt worden war. In Rußland war in den neunziger Jahren des 19. Jahrhunderts eine scharfe Kampagne gegen das Schächten geführt worden; in Norwegen wurde das koschere Schlachten 1930 verboten ${ }^{50}$. In Deutschland reichte die Bewegung gegen das koschere Schlachten bis in die Mitte des 19. Jahrhunderts zurück, und seit 1894 betrieben die deutsche Tierschutzvereine eine Kampagne, mit der die Öffentlichkeit gegen das Schächten ohne Betäubung mobilisiert werden sollte. Auch wenn es jüdischen Organisationen gelang, daß das Thema von der Tagesordnung des Reichstags abgesetzt wurde, so diskutierte die deutsche Öffentlichkeit doch weiterhin diese Frage 51 .

In der zweiten Hälfte der 1920er Jahre waren Rabbiner in Deutschland gezwungen, sich fortwährend mit den gemeinsamen Angriffen der Tierschutzvereine und der Nationalsozialisten auf das Schächten auseinanderzusetzen ${ }^{52}$. Die Gegner des Schächtens behaupteten, das koschere Schlachten sei nicht „human“, also nicht tierfreundlich. Die Rabbiner wandten dagegen ein, gerade diese Art des Schlachtens sei äußerst tierfreundlich. Die Rabbiner ließen sich auf das Argument der Humanität im wesentlichen aus taktischen Gründen ein, sie kämpften letztlich um das Recht, ein religiöses Gebot, eine Mitzwa, zu praktizieren.

Einen ersten Erfolg gegen das Schächten erzielten Tierschutzvereine und Nationalsozialisten in den Jahren 1930/31 in Bayern und Braunschweig, als die Landtage dieser Länder Gesetze verabschiedeten, die das Schächten ohne vorherige Betäubung des Schlachtviehs verboten. Nach dem Januar 1933 wurde die Gesetzgebung in diesem Bereich eilig vorangetrieben: Am 22. März 1933 wurde das koschere Schlachten in Sachsen verboten, am 6. April 1933 in Baden und einigen anderen Ländern. Am 21. April 1933 kam es zum Verbot des Schächtens im gesamten Reich. Gefordert wurde eine vorherige Betäubung des Viehs sowie ein völliges Köpfen des Geflügels beim Schächten ${ }^{53}$.

Der Anteil der religiösen Bevölkerung in den deutsch-jüdischen Gemeinden war mit zehn Prozent im Vergleich zum Anteil der orthodoxen Juden in den polnischen Gemeinden relativ gering. Diese religiösen, gesetzestreuen Juden beachteten die religiösen Gebote und insbesondere die Kaschrut-[Speise-]Vorschriften mit größter Genauigkeit und wurden daher durch die Gesetzgebung gegen das Schächten besonders hart getroffen. Selbst wenn man davon ausgeht, daß die nicht-orthodoxe deutsch-jüdische Bevölkerung sich nicht streng an die Kaschrut-Vorschriften hielt,

50 Art. „Shechita“ (hebr.), Ha-Enzyclopaedia ha-ivrit, S. 696-699.

51 Thor, Intellektuclle Bewältigung, S. 75.

52 Ben-Avner, Arbeit gesetzestreuer Rabbiner, S. 149.

53 Walk, Sonderrecht, S. 5, 11, 15. 
so war die Auseinandersetzung um das Schächten schon in der Weimarer Republik ein grundsätzlicher Kampf um die Bewahrung der Rechte der jüdischen Bevölkerung in Deutschland gewesen. Die liberalen jüdischen Gemeinden traten daher der Orthodoxie in dieser Frage zur Seite ${ }^{54}$. Allerdings dürfen Zweifel angemeldet werden, ob liberale Juden ihre Unterstützung des koscheren Schlachtens langfristig aufrechterhalten und sich bereit erklärt hätten, nach dem Verbot des Schächtens auf den Fleischgenuß gänzlich zu verzichten. In den ersten Monaten glaubten deutsche Juden, den Mangel an koscherem Fleisch durch den Import gefrorenen Fleischs aus Dänemark ausgleichen zu können. Da jedoch dieses Fleisch ungleich teurer war, boten derartige Importe langfristig keine Lösung für den Fleischkonsum der jüdischen Bevölkerung in Deutschland55.

Die Reaktion liberal-jüdischer Kreise auf das Schächtverbot blieb insgesamt zurückhaltend. Einen Tag nach dem Verbotserlaß in Sachsen brachte die Zeitung des Central Vereins die Hoffnung zum Ausdruck, es könne zu einem Ausgleich kommen, der auf den vermeintlichen Tierschutz ebenso Rücksicht nehme wie auf den unausweichlichen Gewissenszwang, also die religiöse Kaschrut-Pflicht ${ }^{56}$. Zwei Wochen später meldete die Zeitung dann sogar, nach dem gegenwärtigen Stand der Wissenschaft sei es richtig, die vom jüdische Religionsgesetz gebotene Schlachtform zu verbieten ${ }^{57}$. Möglicherweise war diese lakonische Meldung Ergebnis einer taktischen Entscheidung, sich aus einer Auseinandersetzung herauszuhalten, auf die der Central Verein ohnehin keinen Einfluß zu haben glaubte. Die fehlende Stellungnahmen und das völlige Schweigen, das die Zeitung des Central Vereins sich selbst auch nach der Ausdehnung des Schächtverbots auf das gesamte Reich auferlegt hatte, lassen vermuten, daß der Central Verein die Frage des koscheren Schlachtens in jenen Tagen nicht für vorrangig hielt.

Auch die Jüdische Rundschau, das Presseorgan der deutschen Zionisten, äußerte sich nicht zur Frage des Schächtverbots. In den ersten Monaten des Jahres 1933 hielt es die Zeitung für falsch, sich zu der entsprechenden Gesetzgebung zu äußern oder deren Auswirkungen zu erörtern. Anfang April 1933 kam die Zeitung ein einziges Mal der Bitte der Vereinigung der traditionell-gesetzestreuen und orthodoxen Rabbiner Deutschlands nach und veröffentlichte einen Aufruf, in dem sich die Rabbiner unter anderem über die Gleichgültigkeit beklagten, die die Bevölkerung der Bewahrung der Kaschrut-Vorschriften entgegenbringe. Außerdem wurde die breite Öffentlichkeit aufgerufen, in dem Verbot des Schächtens in einigen deutschen Städten eine Gelegenheit zu sehen, ein stärkeres Interesse an der jüdischen Tradition und insbesondere am Schächten zu zeigen, denn einen leiderprobten Juden könne auch der Verzicht auf den Fleischgenuß nicht in seiner Gottestreue wankend machen ${ }^{58}$. Dieser Aufruf wurde ohne Kommentar oder Erklärung von seiten der Redaktion gedruckt. Damit gestand die Jüdische Rundschau letztlich ihr Desinteresse an dieser Frage offen ein.

54 Thor, Intellektuelle Bewältigung, S. 75. So veröffentlichte Rabbiner Unna in der Zeitung des Central Vereins 1931 einen Artikel zugunsten des Schächtens. Ben-Avner, Arbeit gesetzcstreuer Rabbiner, S. 150.

55 Der Israelit, Koscher-Flcisch aus Dänemark, Nr. 35, 31. 8. 1933.

56 C.V.Z., Nr. 12, 23. 3. 1933.

57 C.V.Z., Nr. 14, 6. 4. 1933.

$58 \mathrm{JR}$, Eine Kundgebung orthodoxer Rabbiner, Nr. 28/29, 7. 4. 1933. 
So blieb die deutsch-jüdische Orthodoxie in ihrem Kampf gegen das Schächtverbot in Deutschland letztlich allein. Sie versuchte in ihrer Argumentation an das deutsche Eigeninteresse $\mathrm{zu}$ appellieren. In diesem Sinne betonte die Zeitung der deutschen Orthodoxie, Der Israelit, den traditionsverbundenen Charakter des politischen Umbruchs in Deutschland und versuchte, eine Verbindung zum Traditionsbewußtsein an sich herzustellen. Das Schächtverbot, so argumentierte die Zeitung, schwäche die traditionellen Elemente der jüdischen Gesellschaft zugunsten jener extremen und bolschewistischen Faktoren, an deren weiterer Entwicklung das nationalsozialistische Regime in Deutschland keinerlei Interesse haben könne ${ }^{59}$. Auf der anderen Seite widersetzte sich die jüdische Orthodoxie in Deutschland jenen Rabbinern und jüdischen Kreisen, die angesichts der politischen Umstände die $K a-$ schrut-Vorschriften erleichtern wollten. In liberalen Kreisen herrschte die Meinung, die Unmöglichkeit, koscheres Fleisch zu erwerben, könne als „höhere Gewalt“ im religionsgesetzlich-talmudischen Sinne interpretiert werden, was den Verzehr nicht-koscheren Fleisches gestatte. Als Reaktion hierauf schrieb das überparteiliche Israelitische Familienblatt, die jüdische Religion kenne zwar bestimmte Ausnahmen im Bereich der Kaschrut, die jedoch auf keinen Fall auf die Umstände in Deutschland anzuwenden seien ${ }^{60}$.

Um die Situation richtig zu verstehen, muß man sich in Erinnerung rufen, daß in jener Zeit eine vegetarische Ernährung nicht verbreitet war und der Verzicht auf Fleisch allgemein als gesundheitsgefährdend betrachtet wurde. Das Presseorgan der deutschen Orthodoxie publizierte daher zahlreiche von Ernährungswissenschaftlern verfaßte Artikel, die die breite Öffentlichkeit darüber informieren sollten, daß ein gesunder Mensch keinerlei Risiko einginge, wenn er auf Fleisch in seinem Speiseplan verzichte ${ }^{61}$. Außerdem versuchte das Blatt, jüdischen Hausfrauen mit Rezepten für die fleischlose Küche zur Seite zu stehen ${ }^{62}$. Die orthodoxe Führung mußte darüber hinaus jedoch nach Lösungen suchen, die die Fleischversorgung unter den gegebenen Bedingungen längerfristig sicherstellten. Das Israelitische Familienblatt berichete über Rabbinerausschüsse, die verschiedene chemische Methoden der Tierschlachtung und ihre Eignung für den Gebrauch durch die jüdische Öffentlichkeit im Hinblick auf die religionsgesetzlichen Vorschriften und Fragen der Gesundheit prüften 63 . Auch Der Israelit ließ seine Leser wissen, es müßten Wege gefunden werden, die den staatlichen Gesetzen wie den Bestimmungen des Religionsgesetzes gleichermaßen Rechnung trügen. Allerdings betonte die Zeitung im gleichen Atemzug, es bestehe wenig Hoffnung auf eine derartige Lösung64. Im Lauf der Zeit wuchs die öffentliche Unzufriedenheit über die, so stellte sich zunehmend heraus, schleppende und mißliche Behandlung der Frage und die vergebliche Suche nach alternativen Lösungen. Die Führung der Vereinigung der traditionell-gesetzes-

59 Der Israelit, Um die Schechita, Nr. 12, 23. 3. 1933.

60 Israelitisches Familienblatt, Nr. 15, 13. 4. 1933.

61 Der Israelit, Auch Fischflcisch ist Fleisch, Nr. 16, 21. 4. 1933; Schächtverbot und neue Wege für Lebensreform, Nr. 20, 18. 5. 1933.

62 Zum Beispiel: Der Israelit, Kochrezepte für die fleischlose Küchc (Mitgeteilt von der jüdischen Haushaltungsschule Frankfurt a. M.), Nr. 18, 4. 5. 1933.

63 Israelitisches Familienblatt, Der Stand der Schächtfrage, Nr. 23, 8. 5. 1933.

${ }^{64}$ Der Israelit, Zur Durchführungsverordnung über die Schlachtung des Geflügels, Nr. 18, 4. 5. 1933. 
treuen und ortbodoxen Rabbiner Deutschlands versuchte sich gegen den Vorwurf zu wehren, sie unternehme nicht genug, um das Problem zu lösen, und betonte, sie würde alles in ihrer Macht Stehende tun, um die Situation zu ändern. Gleichzeitig ging die Vereinigung zum Gegenangriff auf die Kreise über, die die religionsgesetzlichen Vorschriften erleichtern und Lösungsvorschläge unterbreiten wollten, die von keiner rabbinischen Autorität gebilligt werden könnten ${ }^{65}$. Bei dieser Gelegenheit wies das Blatt darauf hin, daß die deutschen Rabbiner bei der Suche nach geeigneten Lösungen mit Rabbinern aus Osteuropa, darunter Rabbiner Chajim Ozer Grudzanski aus Wilna und Rabbiner Jehuda Zirelson aus Kishinev, in Kontakt stünden. Kontakte zwischen deutschen Rabbinern und Rabbiner Grudzanski aus Wilna und Rabbiner Josef Rozen aus Dwinsk hatte es bereits 1932 gegeben, als sich die Frage der Betäubung vor dem Schächten erhoben hatte ${ }^{66}$. Die Rabbiner wollten in gemeinsamen Gesprächen prüfen, ob bestehende Betäubungsmethoden absolut sicher seien und die Bedingung erfüllten, kein Glied oder Organ des Schlachtviehs $\mathrm{zu}$ verletzen und keine inneren Blutungen zu verursachen, die die Kaschrut beeinträchtigen könnten. Dr. Weinberg, ein deutscher Rabbiner, der an entsprechenden Gesprächen teilnahm, war überzeugt, die „Gelehrten des Ostens" würden sich derartigen Lösungen widersetzen. Er sah die Notwendigkeit gegeben, eine gemeinsame, einheitliche, dem Religionsgesetz, der Halacha, entsprechende Entscheidung zu treffen. Eine derartige Lösung sei auch im Hinblick auf das Schächten von Geflügel notwendig: nach dem deutschen Gesetz sei das Schächten nur bei einer Köpfung des Geflügels erlaubt. Da diese Art des Schlachtens von den orthodoxen Juden nicht akzeptiert werde, müsse unbedingt festgestellt werden, daß mit diesem Vorgehen kein halachisches Verbot verletzt werde.

Derartige Beratungen mit osteuropäischen Rabbinern sowie die Berufung auf deren gutachterliche Meinung beruhten auf der Ansicht, daß in solch grundsätzlichen Fragen Entscheidungen erforderlich seien, die für das gesamte Judentum Geltung beanspruchen konnten, eine Entscheidung der Rabbiner in Deutschland allein also nicht ausreiche ${ }^{67}$. Überdies entwickelte die deutsche Orthodoxie nach dem Ersten Weltkrieg eine große Sympathie für das osteuropäische Judentum, seine Rabbiner und seine religiöse Lebensweise ${ }^{68}$. Dies kam unter anderem in einem gewissen Rückzug der deutschen Orthodoxie aus der Gesellschaft zum Ausdruck. Die in der jüdischen Orthodoxie in Deutschland seit Mitte des 19. Jahrhunderts verbreitete Auffassung "Thora we-Derech Eretz" - also eine Verknüpfung von Thora und Allgemeinbildung bzw. allgemein bürgerlichem Verhalten - wurde von einer Wendung nach innen abgelöst. Junge Rabbinerstudenten, die bald darauf in der Führung der deutschen Orthodoxie eine zentrale Rolle spielen sollten, zeigten Ende der zwanziger Jahre eine zunehmende Neigung zum Studium an osteuropäischen Talmudschulen. 1928 berichtete ein Leser des Blattes $\mathrm{Ha}-\mathrm{Ne}$ 'eman, einer orthodoxen Monatszeitschrift für die junge Generation, daß man in letzter Zeit schon in den litauischen Talmudschulen junge Männer aus Aschkenas [Deutschland] treffen

65 Der Israelit, Die Frage der Fleischversorgung, Nr. 22, 30. 5. 1933.

66 Ben-Avner, Arbeit gesetzestreuer Rabbiner, S. 150.

67 Ebenda, S. 151.

68 Thor, Intellektuelle Bewältigung, S. $64 \mathrm{f}$. Thor spricht von der „Selbstverleugnung“ vor der Meinung der "Gelehrten des Ostens"; ebenda. 
könnte, die ihre Universitätsstudien aufgegeben hätten, um sich mit Begeisterung dem Studium der Thora zu widmen ${ }^{69} .1937$ studierten 80 Studenten aus Deutschland an osteuropäischen Talmudschulen. Sie wurden von dem Keren HaTora finanziert, einer Ausbildungsstiftung der Agudat Israel ${ }^{70}$. Auch religiöse Zionisten entschlossen sich bisweilen zu einem Studium in Polen. Der Bund religiöser Pioniere (Bachad) berichtete im November 1935 über sieben seiner Mitglieder, die an der Talmudschule "Mir" in Polen studierten ${ }^{71}$. Unter diesen Umständen war es nur natürlich, daß deutsche Rabbiner eine schwerwiegende Entscheidung im Bereich der Kaschrut nicht fällen wollten, ohne die Meinung der Thoragelehrten aus dem Osten zu berücksichtigen.

Wenn osteuropäische Rabbiner von deutschen Rabbinern angerufen wurden, um eine Lösung für die infolge des Schächtverbots eingetretene Notsituation zu finden, so hatte deren Lösungsvorschlag natürlich die besonderen Lebensumstände des deutschen Judentums und den Charakter der dortigen Gemeinden zu berücksichtigen. Orthodoxe Kreise in Deutschland fürchteten, liberale Juden, die die Mehrheit des deutschen Judentums ausmachten, könnten die Geduld verlieren und nicht auf eine adäquate Lösung warten, sondern unkoscheres Fleisch essen. Diese Befürchtungen wuchsen, als sich im Laufe der Zeit keine wirkliche Lösung des Problems abzeichnete. Die orthodoxen Rabbiner debattierten während der Sommermonate 1933 auf den Seiten des Israelit mit der religiös-liberalen Führung. Sie dementierten die irrtümliche Behauptung, osteuropäische Rabbiner hätten das Schächten mit vorheriger Betäubung gestattet ${ }^{72}$. Die Orthodoxie wandte sich auch gegen die Annahme des liberalen Rabbinerverbandes, der öffentlich bekanntgemacht hatte:

„Im Wesen des liberalen Judentums liegt es, in jeder Zeit die religiöse Vorschrift in Einklang mit der Lebensmöglichkeit zu bringen, um einer gänzlichen Abkehr vom jüdischen Leben vorzubeugen. Die Religion soll eine Religion des Lebens sein. Wo das Leben eines Menschen gefährdet erschien, wurden darum zu allen Zeiten Dispensationen vom Religionsgesetz ausgesprochen."73

Von Monat zu Monat mehrten sich die Anzeichen dafür, daß die Bereitschaft, die Kaschrut-Vorschriften einzuhalten, zurückging. Der liberale Rabbinerverband riet Gläubigen, die eine Gesundheitsgefährdung infolge des Verzichts auf Fleisch fürchteten, es mit dem Gebot der Kaschrut weniger genau zu nehmen. Die Jüdische Gemeinde zu Berlin, die in vorangegangenen Jahren die Beachtung der Kaschrut-Gebote in ihren Institutionen sehr ernst genommen hatte, meldete nun, sie sei gezwungen, älteren und kranken Menschen in ihren Institutionen den Verzehr nicht-koscheren Fleisches zu gestatten, da es sich doch inzwischen um eine Frage von Leben und Tod handele ${ }^{74}$.

Angesichts dieser Sachzwänge mußten die orthodoxen Rabbiner in Deutschland und insbesonders jene in den Einheitsgemeinden mehr tun, als nur das liberale

69 RKI, Ha-Ne'eman, Orthodoxes Monatsblatt für die junge Generation, Monate TishreCheschwan 5688 (1928), D. Bar-Abba, Die Thora im Westen (ein Brief aus der Schweiz) (hebr., HaTorah be-maarav (michtav miSchweiz), 10. Ausgabe, S. 10.

70 CAHJP, Ansbach N1/48, Agudat Israel Israel World Organisation, 1937.

71 RKI, Bachad, Informationsrundschreiben, November 1935.

72 Der Israelit, Die elektrische Betäubung, Nr. 25, 22. 6. 1933.

73 Der Israelit, Liberale Gewissensentlastung, Nr. 27, 6. 7. 1933.

74 Tydur-Baumel, Klärung, S. 158. 
Judentum anzugreifen. Sie waren sich bewußt, daß eine Lösung gefunden werden mußte, die allen Juden gerecht wurde. Angesichts der großen Verantwortung, die mit einer Revision der Kaschrut-Vorschriften verbunden war, rief die Orthodoxie also die rabbinischen Autoritäten in Osteuropa zur Hilfe. In der Frage des Verzehrs von Geflügel konnte ein erleichternder Ausgleich erzielt werden, obwohl die vom deutschen Gesetz verordneten Schlachtvorschriften nicht dem jüdischen Gebrauch entsprachen, andererseits aber auch keine direkte Übertretung des religiösen Gebots darstellten. Die deutschen Rabbiner gestatteten unter Berufung auf die Autorität osteuropäischer Rabbiner den Verzehr von auf diese Weise geschlachtetem Geflüge $1^{75}$. Schwieriger war die Entscheidung im Hinblick auf die Betäubung von Schlachtvieh. Hier lag ein wirklicher Widerspruch zwischen den deutschen Gesetzesvorschriften und dem jüdischen Religionsgesetz vor. Daher suchten die Rabbiner intensiv nach Betäubungsmethoden, die den Geboten der jüdischen Religion entsprachen. Die Betäubung durch einen Schlag auf den Kopf wurde grundsätzlich abgelehnt, da sie zu einer Gehirnblutung des Tieres führt. Die elektrische Betäubung war eine weitere Alternative. Auch wurde die Möglichkeit einer Betäubung nach dem Schnitt der Halsschlagader geprüft. Um diese Fragen zu klären, fuhr Rabbiner Weinberg 1933 zu Beratungsgesprächen mit Rabbinern nach Marienbad, Krakau, Warschau und natürlich nach Wilna ${ }^{76}$.

Auf Empfehlung der zentralen Autorität in Kaschrut-Fragen, des Rabbiners Chajim Ozer Grudzanski aus Wilna, wurden die Gespräche beschleunigt. Ausgangspunkt war die Annahme, daß die halachischen Aspekte gelöst seien, sobald eine Betäubungsmethode gefunden werde, die dem jüdischen Religionsgesetz gerecht werde. Daher wurden die orthodoxen Rabbiner gebeten, ihnen bereits zu diesem Fragenkomplex vorliegende Materialien als Grundlage einer Erörterung des koscheren Schlachtens einzuschicken 77 . Als weitere Basis der Diskussion verfaßte Rabbiner Weinberg eine Broschüre über alle Aspekte der Debatte, die unter den zuständigen Rabbinern verteilt wurde.

Nach Aussagen Rabbiner Weinbergs war sich Grudzanski des allgemeinen Charakters des Konfliktes um das koschere Schlachten bewußt. Weinberg schrieb, jeder deutsche Jude sollte wissen, daß das Problem des Schächtens nicht auf das deutsche Judentum allein zu beschränken sei. Jeder Jude auf der Welt nehme an der Not und dem Leid der deutschen Glaubensbrüder Anteil. Das Problem des Schächtens sei der schicksalshafte Prüfstein der jüdischen Religion. Die schwierige Notsituation, die zu Zugeständnissen führen könnte, welche im Augenblick noch im Rahmen des Möglichen lägen, könnte auch das koschere Schlachten in anderen Ländern gefährden. Rabbiner Grudzanski sei sich dieser Befürchtung bewußt, wüßte jedoch nicht, ob die Rabbiner eine derartige Verantwortung übernehmen könnten. Er sei sich nicht sicher, ob es für das deutsche Judentum gut sei, wenn diese zwar temporäre Entscheidung, wie auch immer sie mit Gottes Hilfe ausfallen möge, dann eventuell als Vorwand dienen werde, das koschere Schlachten in anderen Ländern völlig zu verbieten ${ }^{78}$.

75 Weinberg, Klärende Responsen, S. 370.

76 Weinberg, Klärende Responsen, S. 370 f.

77 YIVO, RG 116, Folder 119.

78 Weinberg, Klärende Responsen, S. 317. 
Am 23. Mai 1933 kamen die beiden zentralen Organisationen des orthodoxen Judentums - die Vereinigung traditionell-gesetzestrener Rabbiner und der Orthodoxe Rabbinerverband, in Berlin zur Beratung zusammen ${ }^{79}$. An dieser Sitzung nahmen die wichtigsten Autoritäten des orthodoxen Judentums in Deutschland teil: u.a. die Rabbiner Esra Munk, Isak Unna, Jakob Horovitz, Jakob Merzbach. Ein Teil der Konferenzteilnehmer beklagte sich, daß die gelehrten Größen im Osten ohne die vorherige Zustimmung des Orthodoxen Rabbinerverbandes konsultiert worden waren. Im wesentlichen drehte sich die Debatte um die Erwartungen, die die deutschen Rabbiner an die Ratschläge der osteuropäischen Rabbiner knüpften ${ }^{80}$. Rabbiner Horovitz schilderte die Verheerung, die das Schächtverbot und die Tatsache, daß bisher kein Hetter [Dispensierung vom religiösen Gebot] gegeben werden konnte, bereits in weiten Kreisen angerichtet habe. Abgesehen davon, daß Tausende von ehedem gesetzestreuen Juden verlorengegangen seien - in einer Zeit, in der das traditionelle Judentum Eroberungen in einem Umfang hatte machen können wie seit langem nicht mehr, drohe die Institution der Schechita, des Schächtens, aus einer ganzen Reihe von Gemeinden zu verschwinden. Viele der Rabbiner hofften, die Gelehrten könnten einen Hetter geben, da sie dem Druck der jüdischen Öffentlichkeit in Deutschland nichts entgegenzusetzen hätten. Anschaulich wurde diese Situation von Horovitz formuliert, der auch seiner Freude darüber Ausdruck gab, $\mathrm{daß}$ „hier alle Jereim [Orthodoxen] zusammengehen" 81 . Gleichzeitig zeigte er sich über den Verdacht entrüstet, Gemeinderabbiner suchten einen Hetter um jeden Preis; es gehe vielmehr darum, einen tragbaren zu finden.

Doch nicht alle Rabbiner teilten diese Anschauung. Rabbiner Unna glaubte z.B., die deutschen Rabbiner könnten auf die osteuropäischen Rabbiner Druck ausüben, um einen Hetter zu erhalten ${ }^{82}$. Auch fürchteten einige orthodoxe Rabbiner, eine Dispensierung im Bereich der Kaschrut könne als ein Sieg des liberalen Judentums verstanden werden und auf andere Lebensbereiche übergreifen ${ }^{83}$. Die Orthodoxie war sich auch des Einflusses bewußt, den Erleichterungen der religiösen Gebote in einem Land auf andere Länder haben könnten, und spürte daher die Notwendigkeit, äußerst behutsam vorzugehen. Ganz gewiß mußte die besondere Situation des deutschen Judentums berücksichtigt werden, für das Zugeständnisse im Bereich der Kaschrut notwendig waren, da es höchst zweifelhaft war, daß die Mehrheit der Juden in Deutschland den Verzicht auf Fleisch noch lange durchhalten und nicht doch auf unkoscheres Fleisch zurückgreifen würde. Über die Auswirkungen dieser $\mathrm{Zu}$ geständnisse konnte kein Konsens erzielt werden. Einige orthodoxe Rabbiner waren überzeugt, die Zustimmung zur Betäubung des Schlachtviehs würde ein Signal für andere Länder sein und die Einhaltung der traditionell-religiösen Schlachtgebote untergraben. Dies würde ähnliche Gesetzgebungen gegen das Schächten auch in anderen Ländern ermöglichen. Da man hoffte, die Nationalsozialisten und ihre antijüdische Gesetzgebung würden bald wieder von der politischen Bühne verschwinden, stellten die Rabbiner die Frage, ob es sich überhaupt lohne, dies zu ris-

79 YIVO, RG 31, Folder 58, S. 3.

80 Ebenda.

81 Ebenda.

82 Ebenda.

83 Thor, Intellektuelle Bewältigung, S. 78. 
kieren, nur um dem deutschen Judentum einen Ausweg aus einer ohnehin nur vorübergehenden Notlage zu eröffnen. Auf der anderen Seite standen Rabbiner, die meinten, Juden könnten die Aktivität der Antisemiten entschärfen und vielleicht zum Verstummen bringen, wenn sie sich mit der Lösung der Betäubung einverstanden erklärten, was für den Bestand der Tradition des koscheren Schlachtens wichtig sein könnte. Da die Rabbiner zu keiner Entscheidung hinsichtlich der Betäubung gelangen konnten, blieb der Fleischverzehr von Tieren, die vor ihrer Schlachtung betäubt worden waren, zunächst weiterhin verboten.

1935 brach die Diskussion erneut auf, als die Führung des Rabbinerverbandes unter Rabbiner Unna einen Aufruf herausgeben wollte, mit der der jüdischen Bevölkerung nochmals erklärt werden sollte, daß der Verzehr von Tieren, die nach der Betäubung geschlachtet wurden, angesichts aller zu jenem Zeitpunkt vorliegenden Erkenntnisse und Prüfungsverfahren absolut verboten sei (Hervorhebung Y.W.). $\mathrm{Da}$ dies gewissermaßen als ein Hinweis auf spätere Zugeständnisse verstanden werden konnte ${ }^{84}$, widersetzte sich Rabbiner Munk von der orthodoxen Austrittsgemeinde in Berlin dieser Formulierung. Er hielt den zögerlichen Hinweis auf mögliche Zugeständnisse für eine Täuschung. Auch zur Lösung dieser Streitfrage suchten die deutschen Rabbiner die Vermittlung Rabbiner Grudzanskis. Noch 1938 wandte sich Rabbiner Unna an diesen und versuchte, den Konflikt und seine Position zur Rolle der religiösen Führung in dieser Notsituation darzulegen. Unna schrieb, man hätte verschiedene Betäubungsarten untersucht, doch alle seien aufgrund verschiedener Hindernisse verworfen worden. Insbesondere Rabbiner Munk aus Berlin und seine Fraktion, deren aufrechte Gesinnung ihm - Unna - gut bekannt sei, verhielten sich einseitig und befürchteten, jegliches Zugeständnis mit Blick auf die Betäubung würde auch in anderen Ländern zu Forderungen nach Betäubung des Schlachtviehs führen. Tatsächlich jedoch könne die Angelegenheit auch anders verstanden werden. Wenn die Antisemiten nämlich sähen, daß Juden nicht unter Druck gesetzt werden könnten, sondern vielmehr einen Ausweg aus der Notsituation wüßten, dann würde der antisemitischen Propaganda Einhalt geboten werden. Abgesehen davon sei die Größe der Verantwortung, die jene zu tragen hätten, die einen Hetter verhinderten, kaum abzuschätzen. Durch Zögern und passives Abwarten machten sie sich schuldig und würden andere in Schuld führen ${ }^{85}$.

Diese Debatte macht deutlich, daß die Grenze zwischen Befürwortern und Gegnern von halachischen Zugeständnissen und Dispensierungen nicht zwischen Deutschland und Osteuropa verlief: deutsch-jüdische Rabbiner wie Munk neigten zu einer genauen Beachtung der Kaschrut--Vorschriften, obwohl sie die dem deutschen Judentum auferlegten Einschränkungen kannten. Rabbiner außerhalb Deutschlands wie Grudzanski suchten nach Wegen, auf denen der gesamten jüdischen Bevölkerung hätte geholfen werden können. Möglicherweise fühlten sich die Rabbiner der orthodoxen Austrittsgemeinden in Deutschland nicht für die Religiosität der gesamten jüdischen Bevölkerung zuständig und wollten nur für ihre eigenen Anhänger Verantwortung übernehmen. Demgegenüber verstanden sich die Rabbiner der Einheitsgemeinden ebenso wie einige Rabbiner in Osteuropa als Bür-

84 Ebenda, S. 84.

85 Fragen Unnas, S. 37. 
gen der gesamten jüdischen Öffentlichkeit und suchten nach einer Lösung, die allen Ansprüchen gerecht werden sollte ${ }^{86}$.

\section{Polen: Polnische Verfassung und Minderheitenrecht}

Versuche, das vom jüdischen Religionsgesetz vorgeschriebene Schlachten zu verbieten, gab es in den 1920er Jahren auch in Polen ${ }^{87} .1923$ brachte eine völkisch-nationale Organisation, die der Endecja, dem rechten Lager der politischen Szene in Polen, zuzurechnen war, einen Gesetzentwurf zum Verbot des koscheren Schlachtens ein. Der Entwurf wurde mit dem Argument begründet, das Schächten diene Juden als Mittel zur ökonomischen Ausbeutung und Kontrolle des Fleischmarktes. Außerdem meinten die Gesetzesinitiatoren, das koschere Schlachten sei nicht tierfreundlich, nicht hygienisch und werde von der jüdischen Religion eigentlich nicht vorgeschrieben. Der Gesetzentwurf wurde dem Parlamentsausschuß für Industrie und Handel wiederholt zur Debatte vorgelegt, wurde aber nie mehr im Parlament erörtert ${ }^{88}$. Den Gegnern des koscheren Schlachtens in Polen schloß sich auch hier 1928 der Tierschutzverband an. Anfang 1935 ergriff der Tierschutzverband noch einmal die Initiative. Er stützte sich auf die Schriften des Priesters Stanislaw Trzeciak, der in dem jüdischen Schlachten eine Tierquälerei sah. Gegen die Argumente, das Schächten stehe im Widerspruch zum Tierschutz oder werde von der Hebräischen Bibel und dem Talmud überhaupt nicht gefordert, ging man auf jüdischer Seite apologetisch vor. Wie einige Jahre zuvor in Deutschland versuchten Juden in Polen nachzuweisen, daß das koschere Schlachten tierfreundlicher sei als andere Schlachtmethoden.

Unter der Regierung Jozef Pilsudskis stand das oppositionelle rechte Lager hinter den Aktionen zum Verbot des koscheren Schlachtens, während die polnische Regierung das Schächten verteidigte. Diese Haltung der polnischen Regierung zeigte sich deutlich in der Auseinandersetzung, die Polen gegen die Anwendung deutschen Rechts auf das Schächten in den Gebieten Oberschlesiens führte ${ }^{89}$. Die Rechte der jüdischen Bevölkerung in Oberschlesien waren in dem Minderheitenvertrag zwischen Polen und Deutschland im Rahmen des Versailler Vertrages festgelegt worden, und Polen hatte die Möglichkeit, die Einführung nationalsozialistischer judenfeindlicher Gesetze zu verhindern. Polen trat schützend für die in Oberschlesien lebenden Juden ein und konnte erfolgreich das Verbot des Schächtens in diesen Gebieten verhindern. Im Zuge des Rechtsruckes in Polen, der die gesamte polnische Politik in den dreißiger Jahren charakterisierte - ein Umstand, auf den wir später noch zurückkommen werden -, verstärkten sich die Versuche, das

86 Hinweise auf die Affinität zwischen der Haltung der Rabbiner und ihrer Zugehörigkeit zur Austrittsorthodoxie oder der Orthodoxie der Einheitsgemeinden bei: Ben-Avner, Arbeit gesetzestreuer Rabbiner, S. $151 \mathrm{f}$.

87 Zwei frühere derartige Versuche fanden 1878 in Warschau auf Initiative des lokalen Tierschutzverbandes und 1914 infolge der Veröffentlichung des Buches von Andrzej Niemojewski „Die jüdische Seele im Lichte des Talmuds“ statt. Beiden Versuchen war keinerlei Erfolg beschieden: Rudnicki, Ritual Slaughter, S. 148.

88 Zur Abwicklung der Gesetzgebung gegen das Schächten in Polen siehe: Teeni, Hitler's Rise, S. $230 \mathrm{ff} . ;$ Melzer, No Way Out, S. $81 \mathrm{ff}$.

89 YVA, 01/21, 25, Dr. Georg Weissmann, Die Durchsetzung des jüdischen Minderheitenrechts. 
Schächten zu verbieten. Mitte 1935 stand das Problem des koscheren Schlachtens trotz der Proteste jüdischer Repräsentanten erneut auf der Tagesordnung des polnischen Städtetages. Gleichzeitig kam es zu lokalen Versuchen, das Schächten in verschiedenen Städten Polens zu verbieten. Dies stand im Widerspruch zum Landesgesetz, das das Schächten gestattete ${ }^{90}$. Unter den gegebenen Umständen konnten die Zusicherungen des polnischen Religionsministers, das jüdische Schlachten sei in Polen nicht bedroht, die Gemüter nicht beruhigen ${ }^{91}$. Ende 1935 war die Wende im Regierungslager jedoch endgültig vollzogen: Durch die Finanzbehörden verpflichtete der polnische Finanzminister alle koscheren Schlachthöfe der jüdischen Gemeinden, Gewerbescheine zu erwerben und regelmäßige Abgaben zu entrichten. Dies war ein schwerer wirtschaftlicher Schlag gegen das jüdische Schlachtwesen. Der Kampf gegen das Schächten, der zunächst eine Angelegenheit rechter Kreise gewesen war, wurde Anfang 1936 in den regulären Gesetzgebungsprozeß einbezogen. Die Sejm-Abgeordnete Janina Prystor, die früher die Regierung und ihre Maßnahmen unterstützt hatte, brachte einen Gesetzentwurf ein, der die Betäubung von Geflügel und Schlachtvieh vor dem Schächten vorschrieb. Dieser Entwurf wurde vom polnischen Parlament am 7. Februar 1936 debattiert. Er enthielt sieben Artikel und orientierte sich in der Formulierung an dem Gesetz zum Verbot des Schächtens, das 1930 in Bayern verabschiedet worden war. Obwohl sich die Regierung der Verabschiedung des Gesetzentwurfes widersetzte, wurde die Debatte zügig geführt. Der Entwurf erhielt eine knappe Mehrheit im Sejm, wurde zur Erörterung in den zuständigen Unterausschuß weitergeleitet und schließlich einen Monat später gegen den Widerstand der Vertreter der jüdischen und der ukrainischen Minderheit verabschiedet ${ }^{92}$. Zuvor bereits hatten verschiedene Stadträte lokale Schächtverbote erlassen. Obwohl stets der Tierschutz als Motiv für Gesetze und Verordnungen gegen das Schächten angeführt wurde, waren die entscheidenderen ökonomischen Beweggründe offensichtlich und allen bekannt ${ }^{93}$. In Polen lag die Zahl der in der Fleischindustrie beschäftigten Juden bei 60000 Personen ${ }^{94}$. Die Versuchung, Juden aus diesem Sektor zu verdrängen, war also relativ groß. Auch der Umstand, daß Schlachtvieh ohne Hörner wie z.B. Schweine, deren Fleisch ohne Betäubung vor dem Schlachten wohlschmeckender sein sollte, von dem Gesetz ausgenommen waren, wies mehr als alles andere auf die hinter diesem Gesetz stehende wirtschaftliche Motivation hin ${ }^{95}$. Ein weiterer Beleg für den ökonomischen Charakter der Gesetzgebung war der Entwurf zum „Milchgesetz“, der während dieser Zeit im Sejm erörtert wurde ${ }^{96}$. Dieses gesundheitspolitisch begründete Milchgesetz sollte die Milchproduktion und den Verkauf von Milch sowie die Verarbeitung von Milchprodukten einer scharfen Kontrolle unterwerfen und die Zuständigkeit für diese Bereiche

90 C.V.Z. Nr. 21, 23. 5. 1935.

91 Ebenda. Die Zeitung berichtete über die Weigerung des polnischen Radios, der Liga gegen die jüdische Scblachtung Sendezeit zu gewähren, da die Liga sich dagegen aussprach, daß entsprechend ein Rabbiner zugunsten der Schächtung sprach.

92 Zum Verlauf der Debatte im Sejm siehe: Gitman, Jewish Problems, S. $114 \mathrm{ff}$.

93 Auch die Forschungsliteratur weist auf den wirtschaftlichen Faktor als wesentliches Motiv für die Gesetzgcbung gegen das Schächten in Polen. Siche z.B.: Gutman, Polish Antisemitism, S. 103; Korzec, Antisemitism, S. 89 f.

94 Melzer, No Way out, S. 86.

95 Hartglas, Kampf polnischer Juden, S. 147.

96 Melzer, No Way out, S. 82. 
dem Landwirtschaftsministerium unterstellen. Praktisch hätte dies zur Verdrängung der jüdischen Kleinbauern aus der Milchproduktion zugunsten der kooperativen Milchbetriebe geführt. Die Verabschiedung des Gesetzes im Sejm und im Senat am 6. Mai 1936 führte dann auch tatsächlich zur Entlassung vieler Juden aus diesem Wirtschaftssektor.

Neben den jüdischen Erwerbstätigen traf das Schächtverbot vor allem den wirtschaftlichen Status der jüdischen Gemeinden. Nach dem alten Gesetz kamen die Gemeinden in den Genuß von steuerlichen Abgaben, die sie im Rahmen ihrer Funktion als Inspektionsbehörde über die Schechita erheben konnten. Dies war eine wichtige und zentrale Einkommensquelle für die Gemeinden. In Zeiten zunehmender wirtschaftlicher Not unter der jüdischen Bevölkerung Polens trafen die neuen Bestimmungen die finanzielle Situation der Gemeinden besonders hart. Sie mußten ihr Dienstleistungsangebot reduzieren, wodurch es nochmals zu einer Verschärfung der wirtschaftlichen Lage der jüdischen Gesamtbevölkerung kam. Diese Auswirkungen waren den Gesetzesinitiatoren sicherlich nicht entgangen ${ }^{97}$.

Das Gesetz zum Verbot des Schächtens in Polen stand in direkter Verbindung mit den politischen Entwicklungen in Deutschland, auch wenn die Motivation in Polen zunächst eine andere war. Über den kontinuierlichen Einfluß der antijüdischen Gesetzgebung in Deutschland auf das Kräfteverhältnis zwischen polnischen Regierungs- und Oppositionskreisen hinaus wurden in der Debatte des Sejm über das Schächten direkte deutsche Einflüsse spürbar. Wie bereits angemerkt, war der Gesetzesantrag der Sejm-Abgeordneten Prystor eine exakte Kopie des bayerischen Gesetzes von 1930. Während die polnische Seite ihre Kampagne für die Erlaubnis des koscheren Schlachtens in Oberschlesien auf polnisches Recht stützte, das das Schächten erlaubte, machte der Sejm vom Gegenteil Gebrauch und griff zu Argumenten, die die deutsche Seite bereits angewendet hatte, u.a. die Ansicht, das jüdische Schlachten sei unmoralisch und verletze die öffentliche Ordnung ${ }^{98}$. Warum, so fragte einer der Sejm-Abgeordneten, müsse Polen hinter zivilisierten Staaten zurückbleiben und das koschere Schlachten erlauben, während andere „zivilisierte“ Staaten wie Deutschland und die Sowjetunion das Schächten seit langem verboten hätten ${ }^{99}$. Auch der polnische Landwirtschaftsminister Juliusz Poniatowski versuchte das Argument der "Zivilisation“ einzusetzen, um polnische Juden zur Annahme des neuen Gesetzes zu bringen. Sich auf nicht bestätigte Informationen stützend, meinte der Minister, „zivilisierte“ jüdische Gemeinden in Deutschland hätten das Gesetz akzeptiert, das eine Betäubung vor dem Schächten fordere, während andere Gemeinden, die die Betäubung als Methode nicht übernehmen wollten, teures Fleisch aus anderen Ländern importieren müßten. Er brachte die Hoffnung zum Ausdruck, die „zivilisierten“ Gemeinden in Polen würden die Betäubungsmethode akzeptieren und im Laufe der Zeit das polnische Judentum insgesamt beein-

97 Viele Publikationen des Jahres 1935 behaupteten u.a., aufgrund des Monopols der jüdischen Schlachtung auf dem polnischen Fleischmarkt seien die Polen gezwungen, teures Fleisch zu kaufen und somit die jüdischen Gemeinden zu subventionieren. Dieses Argument begegnet in den Ausführungen des Senators Dobaziewsky im Sejm am 27.3. 1936; siehe Melzer, No Way out, S. 81.

98 Gitman, Jewish Problems, S. $122 \mathrm{ff}$.

99 Ebenda, S. 136. 
flussen ${ }^{100}$. Wie schon erwähnt, war das Gesetz zur Einschränkung des Schächtens in Polen im wesentlichen wirtschaftlich motiviert. Es sollte Juden aus dem Sektor der Fleischindustrie verdrängen. Das Gesetz der Abgeordneten Prystor wollte das koschere Schlachten in Polen dagegen gänzlich verbieten. Ein derartiges Verbot stand im Widerspruch zur polnischen Verfassung, die die religiösen Bedürfnisse der nationalen Minderheiten in Polen sicherte. Daher schränkte das Gesetz in seiner endgültigen, von Sejm und Senat am 27. März 1936 verabschiedeten Form das koschere Schlachten ein, verbot es jedoch nicht vollständig. Nur in Orten, in denen der jüdische Bevölkerungsanteil niedriger als drei Prozent war, wurde ein absolutes Verbot verhängt ${ }^{101}$. Das Gesetz sollte am 1. Januar 1937 in Kraft treten.

Doch auch in seiner reduzierten Fassung wirkte sich das Gesetz negativ auf die wirtschaftliche Situation der Juden in Polen aus. Es zog eine Reihe von umfassenden Veränderungen innerjüdischer Politik nach sich. Ganz besonders schwierig war die Situation für die Agudat Israel, die das orthodoxe Judentum repräsentierte. Traditionell vertrat die Agudat Israel eine regierungsfreundliche Position. Bei den Parlamentswahlen 1935 hatten ihre Repräsentanten auf der Liste der Sanacja, also des polnischen Regierungslagers, gestanden. Aus diesem Grunde fiel es der Agudat Israel schwer, später eine gegen die Regierung gerichtete Position zu beziehen. Und obwohl es den Anschein hatte, daß die Agudat Israel sich nicht mit dem Schächtverbot abfinden könne, verhielt sie sich eher zögernd. Rabbiner Chajim Ozer Grudzanski aus Wilna, eine zentrale Persönlichkeit in der Agudat Israel in Polen und in der Debatte mit der deutschen Orthodoxie um das Schächtverbot in Deutschland, wollte ein weltweites Fasten ausrufen lassen, mußte diese Idee jedoch angesichts der unklaren politischen Verhältnisse zugunsten eines behutsameren Verhaltens aufgeben ${ }^{102}$. Zögern und Zurückhaltung der Agudat Israel schwächten ihren politischen Status insgesamt. Viele Juden sahen in der Politik der polnischen Regierung ein Zeichen für das Versagen der von der Agudat Israel vertretenen Position, die trotz ihrer traditionellen Regierungsnähe das Gesetz gegen das Schächten nicht hatte verhindern können ${ }^{103}$. Der Bund, die jüdische sozialistische Partei, jedoch konnte seine Machtposition ausbauen, da er aktiv für die jüdische Bevölkerung eintrat, deren Beschäftigung in der Fleischindustrie gefährdet war ${ }^{104}$.

Der Kampf der polnischen Juden um das koschere Schlachten wurde auf allen Ebenen geführt. Die jüdischen Parteien nutzten in hohem Maße das politische System Polens, nahmen Anteil an der parlamentarischen Debatte und kämpften allgemein um die Gunst der öffentlichen Meinung ${ }^{105}$. Die jüdischen Sejm-Abgeordneten Rubinstein, Sommerstein und Mincberg sowie die jüdischen Senatoren Moshe Schorr und Jacub Trockenheim widersetzten sich heftig den Argumenten, die für

100 Ebenda, S. 118.

101 Hierbei handelte es sich im wesentlichen um Orte in den westlichen Provinzen Polens, in denen die deutschen Abgeordneten mit Gegnern der Schächtung kooperierten; Teeni, Hitler's Rise, S. 90.

102 Melzer, No Way out, S. 86. - Rabbiner Chajim Ozer Grudzanski sah in dem Verbot der Schächtung einen Beleg dafür, daß die Sanacja versuche, mit der Endecja im Judenhaß zu konkurrieren; Mendelsohn, Dilemma of Jewish Politics.

103 Bacon, Agudat Israel, S. 33.

104 Brumberg, Bund, S. 85.

105 Siehe dazu: Melzer, No Way out, S. $86 \mathrm{ff}$. 
das Schächtverbot angeführt wurden, und versuchten, die wahren Gründe hinter dem Gesetzentwurf aufzudecken. Sie verständigten sich auch mit den Vertretern der ukrainischen Minderheit und konnten mit ihnen einen Konsens erzielen, gemeinsam gegen das Gesetz vorzugehen. Allgemein rechnete man mit der Mobilisierung der öffentlichen Meinung der Juden in aller Welt gegen die gesetzlichen Einschränkungen des Schächtens, wie sie sich in den Interventionen der World Federation of Polish Jews, des American Jewish Joint Distribution Committee, des British Board of Deputies und der English-Jewish Association bei polnischen Regierungsstellen ausdrückte. Neben diesen nach außen gerichteten Bemühungen wurde die polnischjüdische Öffentlichkeit mobilisiert. Rabbinerkonferenzen wurden durchgeführt, man rief zum Fasten auf, gründete das Komitee zum Schutze des koscheren Schlachtens und versorgte Regierungstellen mit Informationsmaterial oder führte Pressekonferenzen durch.

Als das polnische Parlament über das Gesetz zum Verbot des Schächtens in Polen debattierte, waren deutschen Juden in ihrem Land bereits seit drei Jahren zu diskriminierten Bürgern geworden. Die „Nürnberger Rassengesetze“ vom September 1935 hatten die bereits praktisch vollzogenen Diskriminierungsmaßnahmen gegen die jüdische Bevölkerung auf eine umfassende gesetzliche Basis gestellt und zusammen mit früheren Gesetzen den Status der Juden in Deutschland als Personen mit minderen Rechten festgesetzt ${ }^{106}$. Die Reaktion deutscher Juden auf das Schächtverbot in Polen sollte daher im Hinblick auf die Frage ihres Status im Deutschen Reich betrachtet werden. Es wurde bereits darauf hingewiesen, daß das Verbot des koscheren Schlachtens in Deutschland im Frühjahr 1933 im wesentlichen das religiöse Judentum traf und nahezu keine Reaktion unter der Mehrheit des deutschen Judentums hervorrief. Demgegenüber wurde das Verbot des Schächtens in Polen zu einem zentralen Thema in den Zeitungen des deutschen Judentums, ein Umstand, der zweifellos auf einen Wertewandel unter deutschen Juden schließen läßt. Die Reaktion deutscher Juden zeigt, wie stark sie mit den politischen Verhältnissen der jüdischen Bevölkerung in Polen vertraut waren und welche Aspekte sie mit Blick auf die Diskriminierung der Juden in Polen wirklich beschäftigten. Außerdem war die Reaktion auf die Ereignisse in Polen unter den gegebenen politischen Umständen ein Weg, auch zu den politischen Entwicklungen in Deutschland indirekt Stellung zu nehmen.

Die deutsch-jüdische Orthodoxie verfolgte gespannt die Auswirkungen der Gesetzgebung in Polen auf die jüdischen Gemeinden und das religiöse Leben. Die Zeitung der deutsch-jüdischen Orthodoxie, Der Israelit, berichtete Anfang Januar 1936 über ein Delegationstreffen der Agudat Israel in Polen mit dem polnischen Innenminister, auf dem die Position des Ministers zum Schächten beeinflußt werden sollte ${ }^{107}$. Zu diesem Zeitpunkt war der Gesetzentwurf zum Verbot des Schächtens noch nicht im Parlament debattiert worden. Jedoch war es in einigen Städten Polens bereits zu einem praktischen Verbot des Schächtens gekommen. Die Zeitung schrieb beispielweise umfassend über die Situation der jüdischen Gemeinde in Lodz ${ }^{108}$. Noch ein weiteres Mal berichtete die Zeitung in jenem Monat über die Ge-

106 Friedländer, Nazi-Germany, Bd. 1, S. $139 \mathrm{ff}$.

107 Der Israelit, Eine Aguda-Delegation bei der polnischen Regierung, Nr. 1, 3. 1. 1936.

108 Der Israelit, Vom Lodzer Kehilarat, Nr. 3, 16. 1. 1936. 
meinde in Lodz, nun jedoch über die Rabbinerwahl109. Die Zeitung kritisierte das Wahlverfahren, das in vielen jüdischen Gemeinden in Polen zur Anwendung gelangte, darunter auch in der Warschauer Gemeinde. Dieses Wahlverfahren führe zur Wahl von Rabbinern, die für ihre Kenntnisse im Religionsgesetz bekannt seien, sich jedoch als ungeeignet für die politische Praxis erwiesen. Das orthodoxe Blatt meinte, der geistige Führer einer Gemeinde wie Lodz müsse natürlich profanes Wissen besitzen und über das politische Geschehen, soweit es die Juden betreffe und alles politische Geschehen in Polen greife mehr oder weniger in die Schicksale der dreieinhalb Millionen Juden ein -, genau Bescheid wissen. Die deutsche Orthodoxie hielt in ihrem Glauben an die Verbindung von Thora und Allgemeinbildung seit Generationen an der Anschauung fest, traditionell-religiöses Wissen allein reiche nicht aus. Vielmehr müsse man sich umfassende allgemeine Kenntnisse erwerben. Daher dürfen entsprechende Äußerungen der orthodoxen Zeitung nicht überraschen. Doch die politische Rolle, die der Orthodoxie in Polen zugesprochen wird, läßt auf ein vertieftes politisches Bewußtsein in orthodoxen Kreisen in Deutschland schließen. Die Reaktion spricht für einen radikalen Wandel der deutschen Orthodoxie im Hinblick auf ihre politische Rolle. Religiöses Wissen, so die Zeitung, reiche nicht aus; oberste Priorität müsse das Wissen um das politische Geschehen erhalten, das Wissen, wie der Kampf um die Bewahrung der Rechte effektiv zu führen sei. Die Kritik, die Der Israelit in diesem Zusammenhang anbrachte, hatte ihre Wurzeln in der orthodoxen Tradition eines Samson Rafael Hirsch, der dem Wissen der rabbinischen Autoritäten über die Welt jenseits der Thora oberste Bedeutung zumaß. Der politische Akzent allerdings, den die Zeitung nun setzte, war ein Resultat der neuen Gegebenheiten nach 1933.

In den folgenden Monaten berichtete Der Israelit regelmäßig über die Entwicklung der Gesetzgebung in Polen ${ }^{110}$. Die Diskussion um das Schächten sorgte in der Zeitung für Schlagzeilen, wobei der besondere Akzent auf dem Bemühen der Agudat Israel in Polen und aller Welt lag, das Schächtverbot zu verhindern ${ }^{111}$. Umfangreiche Aufmerksamkeit widmete Der Israelit auch dem Schicksal der Gemeinden, insbesondere der Frage, ob die finanzielle Haupteinnahmequelle der Gemeinden beeinträchtigt werde. In diesem Zusammenhang erörterte die Zeitung die wirtschaftliche Bedeutung des Schächtens im Rahmen des Gemeindeetats und führte Berichte aus einzelnen Gemeinden an, in denen das Schächten untersagt worden war $^{112}$. Die Aufmerksamkeit, die die Zeitung einzelnen Gemeinden zuteil werden ließ, wurzelte in ihrer religiösen Aufassung: Es ging nicht um die grundsätzliche Frage der Religions- und Gewissensfreiheit, sondern um wesentliche Aspekte der

109 Der Israelit, Zur Rabbinerwahl in Lodz, Nr. 5, 30. 1. 1936.

110 Der Israelit, Eine Schächtverbotsgefahr in Polen, Nr. 7, 13. 2. 1936; Der Schächtverbotsantrag in Polen, Die Gefahren des Schächtverbots, Nr. 8, 20. 2. 1936; Der Gesetzentwurf des Schächtverbots vor dem polnischen Sejm, Nr. 9, 27. 2. 1936, und andere Artikel.

111 So wurde über ein Treffen des politischen Sekretärs der Agudat Israel mit dem polnischen Konsul in London berichtet: Der Israelit, Empfang des politischen Sekretärs der Agudat Israel bei der polnischen Gesandtschaft, Nr. 8, 20. 2. 1936; oder über die Bemühungen der Agudat Israel, bei der polnischen Regierung eine Änderung des Gesetzes zu erwirken: Der Israelit, Intervention der Agudat Israel in London, Nr. 10, 5. 3. 1936.

112 Der Israelit, Ein Zwischenspiel in Polen, Nr. 11, 12. 3. 1936; „Nach Annahme des Schächtverbots", Nr. 14/15, 3. 4. 1936; Die Pessach-Unterstützungsaktion der Gemeinde, Nr. 16, 17. 4. 1936. 
religiösen Existenz des Judentums als einer in der Öffentlichkeit ausgeübten Religion. Daher bestand die Zeitung darauf, daß es weder im Hinblick auf die Einkommensquellen der Gemeinden noch unter halachischen Aspekten, nach denen die Kaschrut in jeder einzelnen Gemeinde zu definieren sei, eine Lösung darstelle, Gemeinden gegebenenfalls die Einfuhr von Fleisch aus anderen Gemeinden zu gestatten $^{113}$. Die Berichterstattung über die Gemeinden hob die dramatische Dimension der Angelegenheit hervor und ermöglichte eine kollektive Identifikation. Der umfassende Bericht über das Schächtverbot in Wilna orientierte sich auch und gerade an der zentralen Bedeutung der Gemeinde in Wilna für das jüdische Leben insgesamt ${ }^{114}$. Das Interesse, das die deutsche Orthodoxie für die Ereignisse um das Schächtverbot in Polen zeigte, darf nicht überraschen. Für eine jüdische Zeitung mit religiöser Leserschaft war es nur natürlich, sich mit Aspekten des religiösen Lebens von Juden in aller Welt zu beschäftigen. In diesem Sinne muß die umfassende Berichterstattung auch als eine Fortsetzung der Debatte gesehen werden, die die Zeitung über das Schächtverbot in Deutschland zu führen versucht hatte. Doch in beiden Fällen wurde eine offene Konfrontation vermieden: Der Israelit berichtete über die Reaktionen von verschiedenen Seiten, informierte jedoch insbesondere über mögliche Lösungen angesichts der neuen Einschränkungen und versuchte zwischen diesen Bestimmungen und den Geboten des Religionsgesetzes zu vermitteln.

Eine deutliche Akzentverschiebung war in der Berichterstattung der Zeitung des Central Vereins über das Schächtverbot zu verzeichnen. Hatte der Central Verein 1933 noch relativ zurückhaltend auf die Gesetzgebung in Deutschland reagiert, so gewann die Gesetzgebung gegen das koschere Schlachten in Polen die gesamte Aufmerksamkeit der C.V.-Zeitung. Wie kann dieses nahezu völlige Schweigen des Central Vereins, der wichtigsten Organisation des liberalen deutschen Judentums, zum Schächtverbot 1933 in Deutschland und die detaillierte Berichterstattung über die Einschränkungen des Schächtens in Polen 1936 erklärt werden?

Als 1933 das Schächten in Deutschland verboten wurde, erfolgte dies durch eines der ersten antijüdischen Gesetze. Die zögernde Reaktion des Central Vereins stand sicherlich mit der Schwierigkeit in Zusammenhang, richtig einzuschätzen, wie weit man Kritik üben dürfe, ohne den C.V. und seine Zeitung zu gefährden. Darüber hinaus wurden die Mitglieder des Central Vereins durch das Schächtverbot nicht direkt getroffen. Andere antijüdische $\mathrm{Maßnahmen}$ hatten für $C$.V.-Anhänger schwerwiegendere Auswirkungen. Man denke nur an das „Gesetz zur Wiederherstellung des Berufsbeamtentums" oder den antijüdischen Wirtschaftsboykott bzw. die Gesetze zur Verdrängung von Juden aus den Hochschulen, Verordnungen, die das Leben liberaler Juden stark erschütterten. Demgegenüber war das Schächtverbot von sekundärer Bedeutung. Die Kräftekonstellation zwischen Orthodoxie und liberalem Judentum sowie bestimmte Parallelen zwischen dem jüdischen Liberalismus im religiösen und dem Liberalismus im Sinne einer Zugehörigkeit zum Central Verein machten es der C.V.-Zeitung schwer, bei einem Thema Stellung zu beziehen, das nicht nur von Antisemiten funktionalisiert wurde, sondern auch zwischen orthodoxen und liberalen Rabinern strittig war. Möglicherweise wurde das Schächtverbot von 1933 zu einer Zeit, als deutsche Juden noch Fleisch aus Nachbarländern impor-

113 Der Israelit, Wilna ohne Schechita!, Nr. 10, 5. 3. 1936.

114 Ebenda. 
tieren konnten, überhaupt weniger dramatisch beurteilt als später. Außerdem war das koschere Schlachten ja auch in Ländern mit starker demokratischer Tradition oder demokratischen Systemen wie z.B. in der Schweiz oder in Norwegen schon einige Jahre zuvor verboten worden. Angesichts der ausdrücklich antijüdischen Verordnungen der Nationalsozialisten nach dem Januar 1933 wurde das Schächtverbot nicht als wesentliche Verletzung begriffen. Möglicherweise fiel es dem Central Verein auch schwer, gegen eine Verordnung vorzugehen, die sich als Tierschutz tarnte. Zunächst mußte diese Tierfreundlichkeit als Vorwand entlarvt werden. Dies alles bleibt natürlich im Rahmen bloßer Spekulation. Selbstverständlich kann auch die Möglichkeit nicht ausgeschlossen werden, daß es für das Schweigen der C.V.Zeitung einen viel einfacheren Grund gab: das fehlende Interesse des Vereins am Schächten.

Anders fielen nun die Reaktionen des Central Vereins zum Schächtverbot in Polen aus. Bereits im Februar 1936 meldete sich die C.V.-Zeitung mit einer umfassenden Studie zu dem Gesetzentwurf zu Wort, in der sich die sehr genaue Kenntnis der Zeitung von den Verhältnissen in Polen widerspiegelte ${ }^{115}$. In ihrer Berichterstattung hob sie die Bemühungen polnischer Juden hervor, das Gesetz zu verhindern. In diesem Zusammenhang wurde über eine Rabbinerkonferenz berichtet, an der 70 führende Rabbiner Polens teilgenommen hätten und auf der eine Protestnote gegen den Gesetzentwurf verabschiedet worden sei. Außerdem seien die Gläubigen zum Fasten und zum Fleischverzicht für den kommenden Monat aufgerufen worden, falls der Gesetzentwurf im Sejm eine Mehrheit erlangen sollte. Auch sollten dann öffentliches Fasten und Gebet ausgerufen werden ${ }^{116}$. Die Zeitung zitierte im Detail das Memorandum, das Rabbiner gegen das Gesetz formuliert hatten, und berichtete über taktische Maßnahmen, die zur Fortsetzung des Kampfes beschlossen worden waren. Als einen Monat später das Gesetz im Sejm verabschiedet worden war, beschäftigte sich die C.V.-Zeitung ausführlich mit der Debatte und den Reaktionen darauf ${ }^{117}$. Auf einer ganzen Seite wurde die Diskussion im Sejm referiert, wurden die Gegenreaktionen innerhalb und außerhalb Polens angeführt und die eigentlichen Motive dargelegt. Eine Woche später berichtete die Zeitung über die Debatte im Senat. Insbesondere den Positionen der jüdischen Senatoren räumte das Blatt breiten Raum ein ${ }^{118}$. Senator Trockenheim, so lautete der Bericht, habe darauf hingewiesen, daß polnische Juden dieses Gesetz als eine Beeinträchtigung ihrer bürgerlichen Rechte und daher als verfassungwidrig betrachteten. Senator Schorr habe betont, Juden sähen in dem Gesetz nicht nur eine Einschränkung ihrer Religion, sondern darüber hinaus der jüdischen Ethik mit ihrem Grundsatz der Nächstenliebe. Schorr habe des weiteren erklärt, Juden lebten seit mehr als 800 Jahren in Polen und

115 C.V.Z., Um die Schechita in Polen, Nr. 9, 27. 2. 1936.

116 Die Reaktion polnischer Juden auf das Schächtverbot entsprach im wesentlichen der Reaktion auf das Schächtverbot in Deutschland drei Jahre zuvor. Auch aus diesem Anlaß hatten Juden in Polen Massenversammlungen abgehalten, während Rabbiner eine rein religiöse "Antwort" und eine besonders strikte Einhaltung der religiösen Vorschriften als passende Antwort auf das Verbot gefordert hatten: Der Israelit, "Massenkundgebungen in Polen“, Nr. 17, 27. 4. 1933.

117 C.V.Z., Das polnische Schächtergesetz und seine Folgen, Nr. 13, 26. 3. 1936.

118 C.V.Z., Auch im polnischen Senat, Nr. 14, 2. 4. 1936. 
hätten gleich den Nichtjuden das Recht, bei öffentlichen Entscheidungen mitzureden.

Angesichts der üblichen Haltung des Central Vereins, der sich traditionell mit antisemitischen Ereignissen auseinandersetzte, war es nur selbstverständlich, daß die C.V.-Zeitung zum Diskussionsforum der Ereignisse in Polen wurde. Wir dürfen nicht vergessen, daß zwischen dem Schächtverbot in Deutschland und dem entsprechenden Gesetz in Polen drei Jahre vergangen waren, in denen dem Central Verein sicherlich nicht die wechselseitige Beeinflussung des Antisemitismus in den Ländern Europas und die kontinuierliche Beeinträchtigung des Status der Juden entgangen sein dürften. Der wesentliche Schaden, den die Einschränkung und das Verbot des Schächtens verursachten, lag - einmal abgesehen von den jüdischen Gemeinden - nicht im wirtschaftlichen Bereich. Vielmehr waren diese Maßnahmen ein Indiz dafür, daß der bürgerliche Status der Juden in Polen angegriffen wurde - ein Umstand, der Juden in und außerhalb Polens sehr wohl bewußt war. Als das Schächtverbot in Deutschland erging, war es nicht deutlich, ob dieses Gesetz im deutschen Kontext eine solche Bedeutung hatte. In Polen erfreuten sich Juden 1936 offiziell jedoch noch der Gleichberechtigung. Zur Debatte stand also letztlich deren Einschränkung durch das Schächtverbot. Wenn die C.V.-Zeitung Senator Trockenheims Worte im Senat zitierte, so forderte sie ihre Leser praktisch auf, an der Grundsatzdebatte über die bürgerliche Gleichberechtigung der Juden in Polen teilzunehmen; denn dort war eine derartige Diskussion noch möglich und sinnvoll.

Auf ähnliche Weise reagierte das Presseorgan der deutschen Zionisten, die Jüdische Rundschau. Während diese Zeitung noch 1933 das Schächtverbot in Deutschland ignoriert hatte, widmete sie 1936 dem Gesetz gegen das koschere Schlachten in Polen breiten Raum ${ }^{119}$. Der Korrespondent des Blattes in Polen hatte die weitreichende Bedeutung des Gesetzes gegen das Schächten deutlich erkannt ${ }^{120}$. Er verwies auf die Ironie, die sich hinter der Auseinandersetzung um das Verbot des Schächtens aus Gründen des Tierschutzes zu einer Zeit verbarg, in der in Polen Juden verfolgt wurden, ohne daß offizielle Stellen diesem Treiben Einhalt zu gebieten versuchten. Hervorgehoben wurde in der Jüdischen Rundschau die Absicht der polnischen Regierung, angesichts der Erfolge des oppositionellen Lagers deutlich darzulegen, daß sie gegen Juden durchaus repressiv vorgehen könne. Darüber hinaus, so betonte der Korrespondent, sei die Angelegenheit des koscheren Schlachtens ein Glied in einer langen Kette antijüdischer Maßnahmen in Polen, deren Ziel die Einschränkung der Bürgerrechte und die Auswanderung von Juden aus Polen sei. Von einem Kampf um die Minderheitenrechte jüdischerseits sei längst keine Rede mehr, so schrieb das Blatt. Der Kampf um die bürgerliche und wirtschaftliche Gleichberechtigung verwandele sich in eine verzweifelte Aktion zum Schutz der nackten Existenz. Daher rief das Blatt zur Verteidigung der Lebensrechte polnischer Juden „als autochthoner, seit Jahrhunderten in Polen seßhafter Bevölkerung“ auf. Auch als der Kampf um das Schächtverbot bereits verloren und das entsprechende Gesetz

$119 J R$, Um den Schächtverbots-Antrag, Nr. 16, 25.2. 1936; Die Schechita-Frage in Polen, Nr. 18, 3. 3. 1936; Um das Schächtverbot in Polen, Nr. 19, 19. 3. 1936; Der Kampf um das Schächtverbot, Nr. 20, 1.3. 1936; Die Lage der polnischen Juden, Nr. 21, 13. 3. 1936; Der Kampf um die Schechita, Nr. 22, 17.3. 1936; Polnisches Schächtgesetz angenommen, Nr. 24, 24. 3. 1936, u. a.

$120 \mathrm{JR}$, Der Verzweiflungskampf der polnischen Juden, Nr. 17, 28. 2. 1936. 
im Senat verabschiedet worden war, sahen Jüdische Rundschau und C.V.-Zeitung die eigentliche Verletzung der jüdischen Bevölkerung in dem gleichen Umstand: Zum ersten Mal seit Gründung der Republik Polen waren Juden per Gesetz diskriminiert worden ${ }^{121}$.

In einem weiteren Leitartikel versuchte die Jüdische Rundschau, der Auseinandersetzung um das koschere Schlachten in Polen eine zionistische Richtung zu geben. Das Blatt lenkte die Aufmerksamkeit seiner Leser auf die Problematik („Anormalität") der ökonomischen Struktur der jüdischen Bevölkerung in der Diaspora, wie sie durch das Schächtverbot aufgedeckt werde ${ }^{122}$. Die Zeitung beschäftigte sich mit den Protestaktionen des polnischen Judentums. Die bewundernde Berichterstattung akzentuierte das breite Spektrum der Reaktionen - Fasten, Demonstrationen, Generalstreik, Volksversammlungen und Massengebete. Ebenso hob das Blatt die jüdische Einigkeit im Kampf um das Schächten hervor, die sich in der Zusammenarbeit aller politischen Richtungen des Judentums in Polen im Protest gegen das Gesetz zeigte ${ }^{123}$. Die polnische Judenheit, so hieß es, besinne sich darauf, daß sie ein Dreieinhalbmillionenvolk sei, seit Jahrhunderten im Lande verwurzelt, dem Staate gegenüber loyal, ein produktiver Faktor in der Wirtschaft. Wie aus einer verborgenen Quelle komme die Lebenskraft des Volkes an den Tag124. Ganz deutlich erkennt man in diesen Zeitungsartikeln, wie hoch die breitgefächerten Reaktionen polnischer Juden eingeschätzt wurden: aus ihnen spreche Lebenswille, Mut und Einheit der polnischen Juden.

Die Meldungen in den drei großen jüdischen Zeitungen in Deutschland - der C.V.-Zeitung, dem Israelit und der Jüdischen Rundschau - über die Entwicklungen in Polen entsprachen sich im wesentlichen. Sie konzentrierten sich auf die verschiedenen Phasen der Gesetzgebung, auf die jüdischen, polnischen und internationalen Reaktionen und letztlich auf die Auswirkungen des Gesetzes für die polnischen Juden. Unterschiede in der Berichterstattung machten sich allein in der Akzentsetzung bemerkbar: Das orthodoxe Blatt widmete sich im wesentlichen den Gemeindeaspekten, während die zionistische Jüdische Rundschau und die liberale C.V.-Zeitung sich auf die politischen Konsequenzen und Reaktionen der polnischen Juden konzentrierten.

Der deutsch-jüdische Diskurs über das Schächtverbot in Polen ermöglichte zweifellos eine indirekte Diskussion über den Antisemitismus und das Wesen der bürgerlichen Gleichberechtigung im allgemeinen. Abgesehen davon war es für deutsche Juden aus politischen Gründen nicht ratsam, ihre Situation mit der Lage der polnischen Juden zu vergleichen. Außerhalb Deutschlands konnte dieser Vergleich jedoch vollzogen werden. Herausragende jüdische Persönlichkeiten wie der Zionist und Sprecher des polnischen Judentums Yitzchak Grünbaum sowie Chaim Weizmann, zu jener Zeit Präsident der Zionist World Federation, waren über den direkten Einfluß der Ereignisse in Deutschland auf Polen und andere Länder besorgt, natürlich ganz besonders angesichts der Diskussion um das Schächten. Grünbaum

$121 J R$, Die Annahme des Schächtgesetzes, Nr. 26, 31. 3. 1936.

$122 \mathrm{JR}$, Blick in die Welt, Nr. 21, 13. 3. 1936.

$123 \mathrm{JR}$, Der jüdische Proteststreik, Nr. 23, 2. 3. 1936; Zusammenfassung der Kräfte, Nr. 25, 31. 3. 1936.

$124 \mathrm{JR}$, Eine Manifestation jüdischen Lebenswillens, Nr. 23, 20. 3. 1936. 
war der Überzeugung, die Debatte im Sejm über ein Verbot des koscheren Schlachtens sei symptomatisch und laufe auf eine grundsätzliche Verletzung der bürgerlichen Gleichberechtigung hinaus ${ }^{125}$. In einem Brief an Nahum Goldmann warnte Grünbaum, die Situation in Polen gebe zunehmend Anlaß zur Sorge. Er glaube nicht, daß man die Schechita gänzlich verbieten werde, hege aber keinen Zweifel, daß sie erheblich eingeschränkt werde. Er schätzte die Lage als kritisch ein. Wenn den antisemitischen Tendenzen kein Ende bereitet werde, würden noch schärfere Diskriminierungen folgen und Polen werde dann bald Deutschland gleichen. Er, Grünbaum, denke, man müsse etwas Außergewöhnliches unternehmen. Wenn dieses furchtbare Gesetz nicht aufgehoben werden könne, ginge das polnische Judentum einer ähnlichen Katastrophe wie das deutsche entgegen. Einziger Unterschied sei, daß in Deutschland 600000 Juden lebten, in Polen aber drei Millionen ${ }^{126}$. Ähnliche Befürchtungen brachte auch Chaim Weizmann in einem Brief an Stephen Wise, den Präsidenten des World Jewish Congress, zum Ausdruck, wenn er schrieb, er glaube, die Situation in Polen sei sogar schlimmer als die in Deutschland. Dort habe man es mit einer unkontrollierbaren Bevölkerung zu tun, die gewohnt sei, Pogrome zu veranstalten. Überdies sei die jüdische Gesellschaft größer als in Deutschland, hätte aber weniger Widerstandskraft und sei ohnehin schon zur Hälfte verhungert ${ }^{127}$.

1936 war das Jahr, in dem das Schächtverbot von Sejm und Senat verabschiedet wurde. Das Gesetz zur Einschränkung des kocheren Schlachtens sollte am 1. Januar 1937 in Kraft treten. Dies führte zu einer nochmaligen Wiederaufnahme der Auseinandersetzung um das Schächten in Polen. Die ursprünglichen Initiatoren begnügten sich nicht mit einer teilweisen Einschränkung des koscheren Schlachtens, sondern verlangten nach einem absoluten Schächtverbot. $1937 \mathrm{kam}$ es daher zu einer weiteren kontinuierlichen Reduzierung der erlaubten Schächtquoten. Die Verhältnisse auf dem Fleischmarkt wurden immer undurchsichtiger, während sich die wirtschaftliche Situation der in diesem Sektor Beschäftigten und der jüdischen Gemeinden deutlich verschlechterte ${ }^{128}$. Der Widerhall dieser Entwicklung reichte bis nach Deutschland. Der Israelit berichtete voller Sorge über einen Antrag zur Einschränkung des koscheren Schlachtens in Litauen ${ }^{129}$. „Inzwischen drängen die Verhältnisse in Polen leider zum Heimatwechsel für die Juden", schrieb die Zeitung im Hinblick auf das Inkrafttreten des Schächtverbots in Polen ${ }^{130}$. Das Blatt veröffentlichte auch Berichte über die wirtschaftlichen Auswirkungen des Verbots und die Proteste der jüdischen Bevölkerung Polens. Daneben standen politische Kommentare zur Bedeutung des Verbots ${ }^{131}$. Auch die C.V.-Zeitung setzte ihre Berichterstattung über die weiteren Einschränkungen gegen das Schächten in Polen fort, wobei es sich allerdings um trockene, sachliche Artikel handelte, die sich gänzlich von den

125 Haynt, Yitzchak Grünbaum, Das Schechita-Verbot in Polen (jidd.), 23. 3. 1936.

126 CZA, L 22/201, Grünbaum an Goldmann, 10. 3. 1936.

127 Weizmann an Wise, 27. 2. 1936, bei Melzer, No Way Out, S. 193.

128 Melzer, No Way out, S. 87.

129 Der Israelit, Das ncue Schlachtungsgesetz in Polen in Kraft getreten, Nr. 1, 7. 1. 1937.

130 Der Israelit, Wochenrundschau, Nr. 2, 14. 1. 1937.

131 Der Israelit, Ein Appell der polnischen Rabbiner, Nr. 4, 28. 1. 1937; Neuer Vorstoß der Abgeordneten Prystor im polnischen Sejm, Nr. 5, 4. 2. 1937; Herabsetzung der Koscherfleisch-Quote, Nr. 7, 18. 2. 1937. 
Kampfesrufen gegen das Schächtverbot des Vorjahres unterschieden. Breiten Raum widmete die C.V.-Zeitung hingegen den antisemitischen Vorfällen an polnischen Universitäten sowie der Grundsatzdebatte über den politischen Status der Juden in Polen und dem Versuch der polnischen Regierung, polnische Juden zur Emigration zu zwingen ${ }^{132}$. Ähnlich sah auch die Berichterstattung in der Jüdischen Rundschan aus, in der die weitere Entwicklung der Frage der Schechita oberflächlich und knapp behandelt wurde ${ }^{133}$, während andere Aspekte des polnischen Antisemitismus weiterhin eine detaillierte Darstellung erhielten. Angesichts der zunehmenden allgemeinen Verschlechterung der Situation polnischer Juden wurde das Verbot des koscheren Schlachtens sekundär.

Die Diskussion im Sejm wurde 1938 ein weiteres Mal aufgenommen, als die Forderung nach einem gänzlichen Verbot des Schächtens erneut laut wurde. Die Regierung versuchte, einen Ersatz für ein derartiges Gesetz zu finden, denn sie war sich bewußt, daß das Gesetz im Gegensatz zur bürgerlichen Gleichberechtigung stand, die durch die Verfassung vom April 1935 garantiert wurde. Im Rahmen der erneut aufflammenden Debatte veröffentlichte der polnische Zionist und bekannte Publizist Moshe Kleinbaum (Sneh) einen Artikel, in dem er vor den negativen politischen und ethischen Folgen eines neuerlichen Gesetzes gegen das Schächten warnte ${ }^{134}$. Seiner Meinung nach erschütterte dieses Gesetz das konstitutionelle Prinzip der Gleichberechtigung von Juden in Polen. Bisher sei die jüdische Bevölkerung administrativ diskriminiert worden, ein derartiges Gesetz stelle jedoch eine konstitutionelle Diskriminierung dar.

Die jüdische Öffentlichkeit in Polen setzte ihren konsequenten Kampf um das Schächten auf mehreren Ebenen fort, im Sejm wie in der Weltöffentlichkeit. Das Ziel war, eine einheitliche Reaktion des polnischen Judentums zu formulieren ${ }^{135}$. Die Motivation, sich überhaupt auf diese Auseinandersetzung einzulassen, war unterschiedlich: Die Zionisten versuchten, die bürgerliche Gleichberechtigung der Juden in Polen zu retten; der Bund sorgte sich um die wirtschaftliche Position der jüdischen Bevölkerung, die Agudat Israel war dagegen eher religiös motiviert. Hier wird deutlich, daß sich alle konkurrierenden politischen Kräfte tendentiell einig waren. Entsprechende Möglichkeiten der Zusammenarbeit boten die Initiativen zum Fleischboykott. Doch die politische Zersplitterung und das Fehlen einer zentralen Führung innerhalb des polnischen Judentums erschwerten das gemeinsame Vorgehen gegen die steten, heftigen Angriffe auf das Schächten in den Jahren 1938-1939136.

Die deutsch-jüdische Presse verlor 1938 schließlich gänzlich das Interesse an der Auseinandersetzung um die Kaschrut in Polen. Die Zeitungsberichte wurden lakonisch und beschäftigten sich nicht mehr mit der prinzipiellen Bedeutung des Verbots, sondern verloren sich in technischen Einzelheiten ${ }^{137}$. Der Rückgang des Inter-

132 C.V.Z., Nachrichten aus Polen, Nr.1, 7. 1. 1937; Das Anwachsen des Schächtproblems, Nr. 2, 14. 1. 1937; Ruhige Wochen in Polen, Nr. 10, 11. 3. 1937.

$133 \mathrm{JR}$, Neuer Verstoß gegen Schechita, Nr. 9, 2. 2. 1937.

134 Haynt, Moshe Kleinbaum, Eine jüdische Stimme warnt (jidd.), 25. 3. 1938.

135 Mclzer, No Way out, S. $87 \mathrm{ff}$.

136 Keinesfalls entsprach die Reaktion der jüdischen Öffentlichkeit 1938 dem entschlossenen Kampf der polnischen Juden von 1936; siehe: Rudnicki, Ritual Slaughter, S. 157.

137 Zum Beispiel: C.V.Z., Nr. 13, 31. 3. 1938; Nr. 14, 7. 4. 1938; Nr. 15, 14. 4. 1938; JR, Verbot 
esses hängt mit einer gleichzeitig sich in Deutschland sowie in Polen und anderen osteuropäischen Staaten vollziehenden Entwicklung zusammen, durch die die Frage des koscheren Schlachtens in Polen selbst für polnische Juden zweitrangig wurde: Von großer Bedeutung für deutsche Juden in jenen Monaten war der Umstand, daß die jüdischen Gemeinden in Deutschland im März 1938 ihren Status als Körperschaften des öffentlichen Rechts verloren. Damit wurde die gesetzliche Pflicht zur Zahlung von Gemeindesteuern aufgehoben. Gemeindesteuern waren fortan eine freiwillige Angelegenheit. Diese Maßnahme traf die jüdischen Gemeinden in Deutschland finanziell ins Mark. Gleichzeitig gerieten Leib und Leben polnischer Juden immer mehr in Gefahr, ihre physische Unversehrheit war nicht mehr garantiert. Zudem beschloß die polnische Regierung Ende März 1938, die Staatsbürgerschaft der außerhalb Polens lebenden polnischen Staatsbürger zu überprüfen. Diese Entscheidung sollte für den Status der polnisch-jüdischen Staatsangehörigen im Ausland schicksalhaft werden. Ähnliche antijüdische Maßnahmen erfolgten in Rumänien und Ungarn. Auch hier wurde das Schächten verboten. Doch das war nun nur noch ein Problem unter vielen. Für die deutschen Juden war es zu diesem Zeitpunkt schon so gut wie bedeutungslos.

der Schechita in Polen, Nr. 25, 29. 3. 1938; Schechita-Verbot in Polen tritt noch nicht in Kraft, Nr. 26, 1. 4. 1938; Der Israelit, Völliges Schechitaverbot in Polen, Nr. 13, 31. 3. 1938. 